\title{
A novel queue-aware wireless link adaptation mechanism and its fixed-point analytical model
}

\author{
Onur Ozturk ${ }^{*}$ and Nail Akar
}

\begin{abstract}
A point-to-point (PTP) wireless link is studied that carries long-lived TCP flows and is controlled with active queue management (AQM). A cross-layer queue-aware adaptive modulation and coding (AMC)-based link adaptation (LA) mechanism is proposed for this wireless link to improve the TCP-level throughput relative to the case where AMC decisions are made based solely on the physical layer (PHY) parameters. The proposed simple-to-implement LA mechanism involves the use of an aggressive modulation and coding scheme (MCS) with high spectral efficiency and high block error rates when the queue occupancy exceeds a certain threshold, but otherwise a relatively conservative MCS with lower spectral efficiency and lower block error rates. A fixed-point analytical model is proposed to obtain the aggregate TCP throughput attained at this wireless link and the model is validated by ns-3 simulations. Numerical experimentation with the proposed analytical model applied to an IEEE 802.16-based wireless link demonstrates the effectiveness of the proposed queue-aware LA (QAWLA) mechanism in a wide variety of scenarios including cases where the channel information is imperfect. The impact of the choice of the queue occupancy threshold of QAWLA is extensively studied with respect to the choice of AQM parameters in order to provide engineering guidelines for the provisioning of the wireless link.
\end{abstract}

Keywords: Fixed-point TCP model, Active queue management, Queue-aware link adaptation, IEEE 802.16

\section{Introduction}

Along with the User Datagram Protocol (UDP) whose use has gained momentum with emerging multimedia and P2P applications, the Transmission Control Protocol (TCP) has been one of the most widely used transport protocols for most Internet services such as Web browsing, file transfer, remote login, and recently for video streaming [1-3]. Buffer management for routers carrying TCP traffic is generally based on active queue management (AQM) mechanisms which drop packets before the routers' queues become full [4-8]. With AQM, large queuing delays that could adversely affect the TCP-level throughput are avoided. Moreover, the drop decision is made probabilistically to mitigate flow synchronization problems (also known as the lock-out problem) described in [4]. We refer to [9] for a survey of AQM in both wireline and wireless contexts. In wireless router links,

*Correspondence: ozturk@ee.bilkent.edu.tr Electrical and Electronics Engineering Department, Bilkent University, Bilkent, Ankara, Turkey non-congestion (or wireless) losses arise due to channel errors in addition to congestion losses caused by AQM drops. TCP suffers from wireless losses since it responds to all losses by triggering congestion avoidance algorithms which results in reduced performance on paths with lossy links [10].

While AQM focuses on buffer management addressing the "full-queues" and "lock-out" problems, link adaptation (LA) refers to mechanisms that match the modulation, coding, and other signal and protocol parameters to the conditions on the wireless link [11]. In existing wireless communication standards, a finite set of collection of physical layer (PHY) parameters, called modulation and coding schemes (MCSs) is defined. Each MCS in this set is then associated with an index $m \in\{0,1, \ldots, M-1\}$ with $M$ being the cardinality of this set. An MCS is then used for transmission of an atomic transmission unit, called a block at the PHY. Throughout the paper, we assume that MCSs are indexed such that the MCS with the largest

\section{黛 Springer}

(C) 2015 Ozturk and Akar. Open Access This article is distributed under the terms of the Creative Commons Attribution 4.0 International License (http://creativecommons.org/licenses/by/4.0/), which permits unrestricted use, distribution, and reproduction in any medium, provided you give appropriate credit to the original author(s) and the source, provide a link to the Creative Commons license, and indicate if changes were made. 
index, i.e., $m=M-1$, is the most aggressive MCS in the set with the highest spectral efficiency and highest block error rates. On the other hand, the MCS with the smallest index, i.e., $m=0$, is the most conservative MCS in the same set with the lowest spectral efficiency and again with the lowest block error rates. Typically, one of the key LA mechanisms known as adaptive modulation and coding (AMC) is employed in existing wireless communication standards to choose the best possible MCS as a function of varying channel conditions on the basis of channel state information (CSI) which is representative of the instantaneous condition of the wireless link $[12,13]$. This optimization problem is involved in maximizing the spectral efficiency of the wireless link under certain block error rate constraints. When these constraints are driven by performance requirements of higher layer applications like multimedia [14], Web browsing, and bulk-data transfer [15], the LA problem requires cross-layer handling. Impact of packet loss and delay incurred by queuing at the data link layer is another research topic for cross-layer analysis [16], which is also studied in [17] for TCP traffic.

In this paper, we study a wireless bottleneck link for a number of long-lived TCP traffic flows with AQM buffer management and AMC-based link adaptation, the interplay between these two components being the main topic of study of this paper, with the goal of potentially increasing the total TCP throughput. TCP-Reno has been the most widely implemented TCP variant $[18,19]$ which we study in this paper. The following information on TCPReno is based on the references [20] and [21]. The sender of a TCP-Reno connection declares a packet to be lost either upon a timeout expiry for its acknowledgment (ACK) packet sent by the TCP receiver or upon the reception of three duplicate acknowledgments (DUPACKs) for a preceding packet. The latter case occurs when three out-of-order packets arrive at the receiver which consequently signals the missing packets via DUPACKs. Upon expiration of a timeout, the sender reduces its congestion window $(\mathrm{CW})$ which represents the collection of packets that are allowed to be transmitted back to back without having to wait for their corresponding ACKs, down to the size of a single packet. DUPACKs are reacted more gently than timeouts by most TCP variants considering the network to be on the verge of congestion. As an example, TCP-Reno triggers a fast retransmit mechanism to retransmit the missing packet reported by the DUPACKs and halves its CW. If an ACK is received in return, then the transmission continues where it is left off; otherwise, the same procedure regarding the timeout condition is executed. After a timeout, TCP-Reno enters into a state called "slow start" at which the $\mathrm{CW}$ is incremented by one for each received ACK. In slow start, the CW doubles every round-trip time (RTT) until a threshold is reached at which a transition to another state called "congestion avoidance" occurs. In the congestion avoidance state, the $\mathrm{CW}$ is approximately incremented at each RTT yielding a linear inflation until either another packet loss is experienced or the advertised TCP receive window (RW) limit is reached at the TCP receiver. The RW is essential for the sender in order not to overwhelm the receiver. In addition to TCP-Reno, there are other more recent variants of TCP such as TCP-Vegas, TCP-Compound, and TCP-CUBIC, the latter two designed for networks with large bandwidth-delay product and are currently in use in Windows and Linux operating systems, respectively, [18, 22-24]. However, the exploration of TCP variants other than TCP-Reno is left outside the scope of this paper throughout which TCPReno and TCP are used interchangeably unless otherwise stated.

An aggressive MCS with high block error rates may lead to high packet error rates (PERs) which in turn throttles back the TCP sources as discussed above, potentially leading to a queue with a high service rate but which is occasionally empty. PHY resources would be wasted in this situation when the queue is empty. On the other hand, conservative MCSs result in low PER leading to a situation with non-empty queues but with lower service rates. In this paper, we focus on PER-based LA which attempts to maintain a desired operational PER value by taking the estimated PER and attempting to reduce the gap between the two [25]. A PER-based AMC mechanism driven solely by the PHY parameters, referred to as a traffic-agnostic link adaptation (TAGLA) in this paper and also in [26], may lead to one of the two abovementioned undesirable situations. Target PER of such schemes can only be optimized if the system parameters of interest are precisely known, e.g., the number of contending TCP flows and their RTTs, in addition to the PHY parameters $[26,27]$. However, estimation of such system parameters is highly difficult in practice [28]. As a remedy, we propose the framework of dual-regime wireless link (DRWL) for which the queue occupancy level is taken into consideration in the process of MCS selection, as opposed to using other system parameters that are hard to estimate. Specifically, we use a conservative (aggressive) MCS when the queue occupancy is below (above) a certain threshold in DRWL. Reducing the probability of "empty queues" and hence the link being under-utilized by TCP sources because of wireless packet losses is the rationale behind DRWL. We view DRWL as a general framework which does not produce a unique policy but instead comprises a set of policies out of which we propose one particular subset of policies called queue-aware link adaptation (QAWLA) in our numerical examples. Actually, the 
QAWLA policy attempts to maintain two particular perregime PER values and therefore belongs to the DRWL framework.

Queue-awareness has been extensively studied in the context of wireless scheduling in multi-user wireless communication systems [29-32]. Energy efficiency is another subject of wireless communications systems for which queue-awareness allows joint control of the transmission power and rate for given QoS constraints [3336]. Assuming an error-free point-to-point (PTP) link operating at the channel capacity, the reference [37] devises an optimal power control scheme called joint queue length aware (JQLA) power control for a set of QoS constraints comprising packet drop probability (which occurs due to finite buffer length), maximum delay, and the arrival rate. In a simulation-based study, the authors propose a distributed traffic-aware power control algorithm for multi-hop IEEE 802.11 wireless networks adapting transmission rates to satisfy the network-wide traffic demand [38]. For a fixed signalto-interference-plus-noise ratio (SINR) level, however, a single MCS satisfying a pre-determined bit error rate (BER) is chosen. By disseminating the so-called "virtual buffers" throughout the nodes of a hybrid wired and code division multiple access (CDMA) wireless cellular network with a distributed algorithm, joint transmission power and rate optimization is formulated as a network utility maximization problem which can be solved by the congestion control algorithms of TCP [39]. The so-called jointly optimal congestion control and power control (JOCP) algorithm outlined in the reference [40], on the other hand, iteratively updates the transmission power of each node in a multi-hop wireless network by sharing weighted queuing delay information in a distributed manner assuming TCP-Vegas to be the source of the generated traffic. Convergence of JOCP, however, is not guaranteed for TCP-Reno whose congestion control relies on packet losses rather than delays as with TCP-Vegas. Finally, the presented AMC scheme in the reference [41] for an interference-limited two-hop relay network chooses an MCS based on both the current SINR level and the number of available packets in the transmission queue of the relay node, whichever suggests the minimum, but does not take into account any higher layer traffic such as TCP. To the best of our knowledge, this is the first study employing queue-awareness in AMC decisions to specifically improve TCP-Reno throughput performance.

An analytical expression, known as the "PFTK" formula, is already available for the packet sending rate of a longlived TCP flow as a function of its packet loss rate and RTT [42]. The PFTK formula takes into account both the fast retransmit mechanism of TCP-Reno and the effect of TCP timeout on packet sending rate. Using a fixed- point model, the PFTK formula has been successfully used to approximate the throughput of a long-lived TCP flow, sharing an AQM-controlled wireline link, or feeding into a network of AQM-controlled wireline links along with other long-lived TCP flows [43]. Making use of the well-established PFTK formula, we propose in this paper a fixed-point model of a single AQM-controlled wireless link with AMC decisions being based on the DRWL framework. Our modeling work is substantially different than [43] due to the special behavior at the boundary between the two regimes of interest. In [43], the queue service rate is fixed for all queue occupancies. However, in the current study, not only the queue service rate but also the wireless packet error rate depends on the queue occupancy in a piece-wise continuous manner with a discontinuity at a single boundary point. Such discontinuities lead to scenarios where the boundary point may become the steady-state fluid limit and the conventional fixedpoint model of [43] falls short of modeling discontinuous queue service rates and wireless packet loss rates. For such scenarios, we propose an extended fixed-point analytical model to model TCP throughput in AQMcontrolled wireless links in the current study. The proposed fixed-point analytical model has a computational complexity low enough to enable the exploration of the multi-dimensional problem space spanned by the number of TCP flows, the number of MCSs, and varying signal-tonoise ratio (SNR) levels, which would not be feasible with a study based solely on simulations. However, ns-3 simulations are carried out for a subset of scenarios to validate the proposed model. Existence and uniqueness conditions are presented for the solution of the fixed-point analytical model. Using the findings of the stochastic model, we show that robust TCP-level throughput improvement over TAGLA is attainable by QAWLA in a wide variety of scenarios. We use the same PFTK TCP formula, AQM scheme, and the set of MCSs with the work [26] presenting an analysis of TAGLA and replicate the related content in the current paper for the sake of completeness. Hybrid ARQ (HARQ)/ARQ techniques for which the blocks/packets get to be retransmitted upon loss at the link layer are not considered in this paper and are left for future study.

The paper is organized as follows. In Section 2, we present the general DRWL framework along with the presumed assumptions and the particular QAWLA mechanism we propose for link adaptation. Section 3 presents the fixed-point model for the DRWL framework. In Section 4, traffic and wireless link scenarios used in the numerical experiments are described. Section 5 addresses the validation of the proposed fixed-point model using extensive ns-3 simulations. In Section 6, we provide numerical examples to validate the effectiveness of the proposed QAWLA scheme. We conclude in the final section. 


\section{Dual-regime wireless link}

We envision a PTP wireless link employing AQM which maintains a shorter average queue length than its droptail counterpart. One of the most popular AQM schemes is random early detection (RED) for which an arriving packet is dropped with a probability depending on the average queue occupancy [5]. The RED scheme interprets "the average queue occupancy exceeding a minimum queue threshold denoted by $t h_{\text {min }}$ " as an onset of network congestion and reacts by linearly increasing its drop probability from 0 up to the value $p_{\max }$ until the maximum queue threshold denoted by $t h_{\max }$ is reached. Beyond $t h_{\text {max }}$, the arriving packets are dropped deterministically. The performance of RED is known to be sensitive to the choice of its parameters $p_{\max }$ and $t h_{\max }$ and as a remedy, the so-called gentle variant of RED denoted by GRED has been proposed having a continuous drop probability function $q(x)$ as follows $[44,45]$ :

$$
q(x)= \begin{cases}0, & 0 \leq x<t h_{\text {min }} \\ \frac{x-t h_{\min }}{t h_{\max }-t h_{\min }} p_{\max }, & t h_{\min } \leq x<t h_{\max } \\ p_{\max }+\frac{x-t h_{\max }}{t h_{\max }}\left(1-p_{\max }\right), & t h_{\max } \leq x<2 t h_{\max } \\ 1, & \text { otherwise }\end{cases}
$$

where $x$ denotes the queue occupancy. The drop probability function $q(x)$ in Eq. (1) is illustrated in Fig. 1 by a red line. We use GRED in this paper for buffer management but other AQM schemes could also be employed.

The wireless link is assumed to carry $N$ long-lived TCPReno flows using first-in-first-out (FIFO) scheduling with a common packet size $L$. Each flow $i, i \in\{0,1, \ldots, N-1\}$, is assumed to have a fixed (yet arbitrary) RTT denoted by $R T T_{0, i}$, taking into account the propagation delays of all links on the path of the flow $i$. We assume all flows are bottlenecked at this wireless link and packet losses on other links are assumed to be negligible. We therefore do

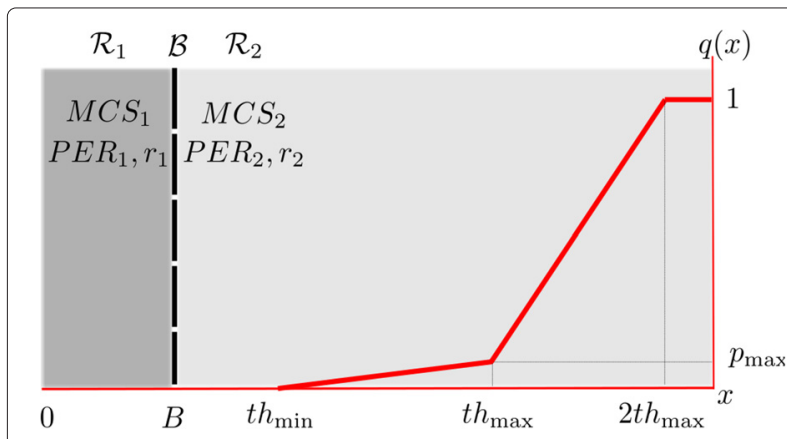

Fig. 1 Illustration of DRWL with the $x$-axis representing the queue occupancy $x$ not attempt to model networks of AQM router links but rather focus on a single AQM bottleneck link in this study. Queuing and transmission delays as well as the error rates of the TCP ACK packets are assumed to be negligibly small and will be ignored by the analytical model assuming TCP ACK prioritization to be employed and enhanced wireless protection to be established at the reverse path of the flows $[46,47]$.

For link adaptation purposes, we introduce in this paper a threshold $B$ and subsequently partition the queue of the wireless link into two different regimes, namely $\mathcal{R}_{1}=$ $[0, B)$ and $\mathcal{R}_{2}=\left(B, 2 t h_{\max }\right)$ along with the boundary $\mathcal{B}=\{B\}$. The queue is served with a transmission rate $r_{1}\left(r_{2}\right)$ with a wireless PER denoted by $P E R_{1}\left(P E R_{2}\right)$ when the buffer occupancy $x$ resides in regime $\mathcal{R}_{1}\left(\mathcal{R}_{2}\right)$ such that $r_{1} \leq r_{2}$ and $P E R_{1} \leq P E R_{2}$. In the abovementioned definition, the strict inequality case is definitely more interesting but we let DRWL to be more general by allowing equalities. We present an overlaid illustration of the proposed DRWL and the GRED AQM scheme in Fig. 1. This DRWL can be generalized to a multi-regime scenario by further partitioning the queue into more than two regimes, but we limit our scope only to DRWL in this paper. The DRWL framework consists of a set of queue-aware link adaptation mechanisms out of which we propose a particular subset of policies next. For this purpose, we consider $M$ different candidate PHY MCSs denoted by $m c s_{m}$ where $m \in\{0,1, \ldots, M-1\}$ that are supported by the wireless link's air interface. When a packet gets to be transmitted, we use a dedicated MCS at each regime, namely $M C S_{1}$ and $M C S_{2}$, when the queue length at the epoch of packet transmission resides in $\mathcal{R}_{1}$ and $\mathcal{R}_{2}$, respectively. If the queue occupancy resides at $\mathcal{B}$, either $M C S_{1}$ or $M C S_{2}$ can be used. When $M C S_{j}$ equals $m c s_{m}$ for regime $j, j \in\{1,2\}$, and for SNR level $s n r_{s}$, $s \in\{0,1, \ldots, S-1\}$, packets are transmitted with a bit rate of $r_{j}=g_{m}$ and errored at the receiver with a probability denoted by $P E R_{j}=$ per $_{m, s}$ where $g_{m}$ is the bit rate of $m c s_{m}$ seen by the link layer and $p e r_{m, s}$ is the PER when $m c s_{m}$ is used at SNR level $s n r_{s}$.

In this paper, we propose a particular DRWL mechanism denoted by $Q A W L A\left(t_{P E R}, H, B\right)$ in terms of three parameters $t h_{P E R}, H$, and $B$. In this PER-based LA mechanism, the MCS with the highest spectral efficiency whose resulting PER denoted by per $_{m, s}$ is such that per $_{m, s}<$ $t h_{P E R}$ for a threshold parameter $t h_{P E R}$ at a particular SNR level $s n r_{s}$, is chosen for regime $\mathcal{R}_{2}$. MCS decision for $\mathcal{R}_{1}$ is given in the same manner with $\mathcal{R}_{2}$, but with a lower threshold $t h_{P E R} / H$ for some $H \geq 1$. It is clear that QAWLA $\left(t_{\text {PER }}, H, B\right)$ belongs to the DRWL framework for the entire range of its parameter set. For the particular QAWLA mechanism with $H=1$, we have $M C S_{1}=M C S_{2}$ irrespective of the queue occupancy at the same channel conditions and is therefore referred to as TAGLA(thPER), 
i.e., traffic agnostic link adaptation. In the next section, we develop a fixed-point analytical model of the generic DRWL by means of which we evaluate the performance of the specific QAWLA scheme to be used in the numerical examples.

\section{Fixed-point analytical model of DRWL}

In line with the majority of the existing work on TCP modeling, we propose to use the so-called PFTK TCP formula which relates the packet sending rate of a TCP flow to the packet loss rate seen by the flow [42]. Let $p, \lambda, L$, and $T_{0}$ denote the packet loss rate, packet sending rate in packets/s, packet size in bits, and the retransmission timeout parameter of a TCP source, respectively. For the timeout parameter, we use

$$
T_{0}=\max \left(T_{0, \min }, R T T+4 \sigma_{R T T}\right),
$$

where $R T T$ and $\sigma_{R T T}$ are the smoothed estimates for the RTT and its standard deviation, respectively, and $T_{0, \min }$ is a minimum limit imposed on the timeout parameter [48]. Let $W_{u}$ denote the random variable associated with the unconstrained window size of the TCP source. Also let $W_{\max }=W / L$ and $b$ denote the maximum window size in units of packets and the number of packets to wait before sending a cumulative ACK packet by the TCP receiver, respectively, where $W$ is the receiver's buffer size. The reference [42] proposes the following identity, known as the PFTK formula, for the TCP sending rate $\lambda$ if the TCP flow faces a packet loss rate $p$ :

$\lambda= \begin{cases}\frac{\frac{1-p}{p}+E\left[W_{u}\right]+\tilde{Q}\left(E\left[W_{u}\right]\right) \frac{1}{1-p}}{R T T\left(\frac{b}{2} E\left[W_{u}\right]+1\right)+\tilde{Q}\left(E\left[W_{u}\right]\right) T_{0} \frac{f(p)}{1-p}}, & E\left[W_{u}\right]<W_{\max } \\ \frac{\frac{1-p}{p}+W_{\max }+\tilde{Q}\left(W_{\max }\right) \frac{1}{1-p}}{R T T\left(\frac{b}{8} W_{\max }+\frac{1-p}{p W_{\max }}+2\right)+\tilde{Q}\left(W_{\max }\right) T_{0} \frac{f(p)}{1-p}}, & \text { otherwise, }\end{cases}$

where

$$
f(p)=1+p+2 p^{2}+4 p^{3}+8 p^{4}+16 p^{5}+32 p^{6},
$$

$\tilde{Q}(w)=\min \left(1, \frac{\left(1-(1-p)^{3}\right)\left(1+(1-p)^{3}\right)\left(1-(1-p)^{(w-3)}\right)}{1-(1-p)^{w}}\right)$,

and

$$
E\left[W_{u}\right]=\frac{2+b}{3 b}+\sqrt{\frac{8(1-p)}{3 b p}+\left(\frac{2+b}{3 b}\right)^{2}} .
$$

Note that Eq. (3) provides a closed-form expression for the TCP sending rate $\lambda$ provided the parameters $p$ and $R T T$ are available. In this study, we assume all TCP flows use the same minimum timeout parameter $T_{0, \min }$ and the term $R T T+4 \sigma_{R T T}$ in (2) is much smaller than $T_{0, \text { min }}$ which yields $T_{0}=T_{0, \mathrm{~min}}$. Exploiting this assumption, we simplify Eq. (3) to be used in sequel as follows:

$$
\lambda= \begin{cases}\frac{1}{R T T P_{1}+P_{2}}, & E\left[W_{u}\right]<W_{\max } \\ \frac{1}{R T T P_{1, c}+P_{2, c}}, & \text { otherwise }\end{cases}
$$

where $P_{1}$ and $P_{2}\left(P_{1, c}\right.$ and $\left.P_{2, c}\right)$ are functions that represent the packet loss rate dependency of the unconstrained (constrained) TCP packet sending rate as defined below:

$$
\begin{aligned}
& P_{1}=\frac{\frac{b}{2} E\left[W_{u}\right]+1}{\frac{1-p}{p}+E\left[W_{u}\right]+\tilde{Q}\left(E\left[W_{u}\right]\right) \frac{1}{1-p}}, \\
& P_{2}=\frac{\tilde{Q}\left(E\left[W_{u}\right]\right) T_{0} \frac{f(p)}{1-p}}{\frac{1-p}{p}+E\left[W_{u}\right]+\tilde{Q}\left(E\left[W_{u}\right]\right) \frac{1}{1-p}}, \\
& P_{1, c}=\frac{\frac{b}{8} W_{\max }+\frac{1-p}{p W_{\max }}+2}{\frac{1-p}{p}+W_{\max }+\tilde{Q}\left(W_{\max }\right) \frac{1}{1-p}},
\end{aligned}
$$

$$
P_{2, c}=\frac{\tilde{Q}\left(W_{\max }\right) T_{0} \frac{f(p)}{1-p}}{\frac{1-p}{p}+W_{\max }+\tilde{Q}\left(W_{\max }\right) \frac{1}{1-p}} .
$$

Since the pairs $\left(r_{1}, P E R_{1}\right)$ and $\left(r_{2}, P E R_{2}\right)$ are not necessarily identical, it may be possible that the combined AQM-AMC policy may push the buffer occupancy from $\mathcal{R}_{1}$ outward to $\mathcal{R}_{2}$ and vice versa, making the boundary $B$ the fixed point. This can be viewed as the queue occupancy hovering in close vicinity of the boundary $B$, visiting both regimes infinitely often but transmitting packets in $\mathcal{R}_{1}$ and $\mathcal{R}_{2}$, with probabilities $(1-\alpha)$ and $\alpha$, respectively, at the steady-state. For higher (lower) values of $\alpha$, a larger ratio of packets admitted into the queue finds the queue in the $\mathcal{R}_{2}\left(\mathcal{R}_{1}\right)$ regime. Consequently, the queue is modeled to be served with a transmission rate of $r(x, \alpha)$ when the queue occupancy is $x$ :

$$
r(x, \alpha)= \begin{cases}r_{1}, & 0 \leq x<B \\ r_{1}(1-\alpha)+r_{2} \alpha, & x=B \\ r_{2}, & B<x<2 t h_{\max }\end{cases}
$$

and the average wireless loss probability, denoted by $\operatorname{PER}(x, \alpha)$, can be written as follows: 


$$
\operatorname{PER}(x, \alpha)= \begin{cases}P E R_{1}, & 0 \leq x<B \\ \frac{P E R_{1} r_{1}(1-\alpha)+P E R_{2} r_{2} \alpha}{r(B, \alpha)}, & x=B \\ P E R_{2}, & B<x<2 t h_{\max }\end{cases}
$$

Note that when $x=B$, the transmission rate and wireless loss probability also depend on the parameter $\alpha$ which needs to be calculated. Based on the philosophy of DRWL, we assume $P E R_{1} \leq P E R_{2}<1$ in which case the TCP receivers get service from both regimes and $r_{1} \leq r_{2}$. Lost packets are not retransmitted and loss events are assumed to be independent and identically distributed (iid) following the Bernoulli wireless loss model. Assuming $\mathrm{AQM}$ and wireless packet losses to be independent from each other, the overall loss probability can then be expressed as

$$
p(x, \alpha)=1-(1-\operatorname{PER}(x, \alpha))(1-q(x)) .
$$

All flows are exposed to a queuing delay $x / r(x, \alpha)$ and a transmission delay $L / r(x, \alpha)$ at the router. Without loss of generality, we let $D_{F}$ account for the one-way framing and processing delays. Moreover, $D_{F}$ is multiplied by a factor of two in order to cover both forward and reverse (TCP ACK messages) path delays. Taking into account the propagation delays of all links on the path of the flow $i$, we have the following expression for the overall RTT of flow $i$ :

$$
R T T_{i}(x, \alpha)=R T T_{0, i}+2 D_{F}+x / r(x, \alpha)+L / r(x, \alpha) .
$$

We take similar steps with [43] in relating the PFTK TCP model given in (3) with the queue occupancy $x$ but also considering the dual-regime nature of the queue. The overall rate of bits that are admitted into the queue denoted by $\kappa(x, \alpha)$ can then be written as

$$
\kappa(x, \alpha)=L(1-q(x)) \sum_{i=0}^{N-1} \lambda_{i}(x, \alpha)
$$

where $\lambda_{i}(x, \alpha)$ is the packet sending rate of flow $i$ when the queue takes the value $x$ and we propose to use the PFTK TCP formula (3) to write $\lambda_{i}(x, \alpha)$ with $R T T$ and $p$ being replaced with $R T T_{i}(x, \alpha)$ and $p(x, \alpha)$, respectively. The goal of the fixed-point model is to find the steadystate buffer occupancy denoted by $x^{\star}$ which can be viewed as an approximation to the mean queue occupancy in the actual system. Assuming that the queue has a steady-state solution at $(x, \alpha)=\left(x^{\star}, \alpha^{\star}\right)$, the following fixed-point identity should hold:

$$
\kappa\left(x^{\star}, \alpha^{\star}\right) \begin{cases}=r\left(x^{\star}, \alpha^{\star}\right), & x^{\star}>0 \\ <r_{1}, & x^{\star}=0 .\end{cases}
$$

Given the steady-state solution $\left(x^{\star}, \alpha^{\star}\right)$, the aggregate TCP throughput of the system denoted by $T$ is given by the following identity:

$$
T=\left(1-\operatorname{PER}\left(x^{\star}, \alpha^{\star}\right)\right) \kappa\left(x^{\star}, \alpha^{\star}\right) .
$$

Next, we take the preliminary steps leading to the proof for existence and uniqueness of the solution to DRWL for $r_{1}<r_{2}$ and $P E R_{1}<P E R_{2}$. For the particular case when $r_{1}=r_{2}$ and $P E R_{1}=P E R_{2}$, we refer the reader to [43]. Recalling Eq. (7) and replacing the terms $P_{1}, P_{2}, P_{1, c}$ and $P_{2, c}$ therein with their $x$ and $\alpha$ dependent counterparts $P_{1}(x, \alpha), P_{2}(x, \alpha), P_{1, c}(x, \alpha)$ and $P_{2, c}(x, \alpha)$, respectively, as well as the RTT term with its $x$ and $\alpha$ dependent perflow counterpart $R T T_{i}(x, \alpha)$, we express the per-flow TCP sending rate as follows:

$$
\lambda_{i}(x, \alpha)= \begin{cases}\frac{1}{R T T_{i}(x, \alpha) P_{1}(x, \alpha)+P_{2}(x, \alpha)}, & E\left[W_{u}\right]<W_{\max } \\ \frac{1}{R T T_{i}(x, \alpha) P_{1, c}(x, \alpha)+P_{2, c}(x, \alpha)}, & \text { otherwise. }\end{cases}
$$

Since TCP packet sending rate is a monotonically decreasing (MD) function of packet loss rate regardless of the RTT, the functions $P_{1}(\cdot)$ and $P_{2}(\cdot)$, and additionally $P_{1, c}(\cdot)$ and $P_{2, c}(\cdot)$, are monotonically non-decreasing (MND) in $x$, given that $p(x, \alpha)$ in (14) is MND. The MND property of $p(x, \alpha)$ is implied by the inequality $P E R_{1}<$ $P E R_{2}$ inherited from DRWL and the fact that $q(\cdot)$ is MND. Without loss of generality, we provide the proof for existence and uniqueness only for the unconstrained TCP packet sending rate using the functions $P_{1}(\cdot)$ and $P_{2}(\cdot)$. The aggregate bit arrival rate to the queue can then be written using (19) as:

$$
\begin{aligned}
L \sum_{i=0}^{N-1} \lambda_{i}(x, \alpha) & =\sum_{i=0}^{N-1} \frac{L}{\left(R T T_{0, i}+2 D_{F}+\frac{x+L}{r(x, \alpha)}\right) P_{1}(x, \alpha)+P_{2}(x, \alpha)} \\
& =\frac{r(x, \alpha)}{G(x, \alpha)},
\end{aligned}
$$

where $G(x, \alpha)$ equals

$$
\left(\sum_{i=0}^{N-1} \frac{L}{\left(\left(R T T_{0, i}+2 D_{F}\right) r(x, \alpha)+x+L\right) P_{1}(x, \alpha)+P_{2}(x, \alpha) r(x, \alpha)}\right)^{-1},
$$

and is a strictly positive monotonically increasing (MI) function provided that $r(x, \alpha)$ in (12) is MND which is true since $r_{1}<r_{2}$. With these definitions, the identity (17) can further be simplified as follows: 


$$
G\left(x^{\star}, \alpha^{\star}\right) \begin{cases}=1-q\left(x^{\star}\right), & x^{\star}>0 \\ >1, & x^{\star}=0\end{cases}
$$

which is the main identity we refer to in this paper that needs to be satisfied at the steady-state. Next, we present the proof for existence and uniqueness of a solution to DRWL in Theorems 3.1 and 3.2, for both $\mathcal{R}_{j}, j \in\{1,2\}$ and $\mathcal{B}$, respectively.

Theorem 3.1. There exists a unique solution $x^{\star}$ to the dual-regime queue provided that $r_{1}<r_{2}, P E R_{1}<P E R_{2}$, $\kappa\left(2 t h_{\max }, \alpha\right)<r_{2}$ and $q(x)$ is an MND continuous function of $x$.

Proof 1. The condition $\kappa\left(2 t h_{\max }, \alpha\right)<r_{2}$ guarantees that full-queue never occurs. It is clear that the identity $G\left(2 t h_{\max }, \alpha\right)>1-q\left(2 t h_{\max }\right)=0$ holds. Since the function $1-q(x)$ is monotonically non-increasing and continuous, $G(x, \alpha)$ is monotonically increasing in $x$ given that $r_{1}<r_{2}$ and $P E R_{1}<P E R_{2}$; either $G\left(x^{\star}, \alpha^{\star}\right)=1-q\left(x^{\star}\right)$ for some $\left(x^{\star}, \alpha^{\star}\right)$ where $x^{\star} \in\left(0,2 t h_{\max }\right]$ and $\alpha^{\star} \in[0,1]$; or $G(0, \alpha)>$ $1-q(0)=1$ for $x^{\star}=0$ must be satisfied. Uniqueness of $x^{\star}$ follows from $G(x, \alpha)$ being monotonically increasing and $1-q(x)$ being monotonically non-increasing.

Theorem 3.2. Provided $r_{1}<r_{2}$ and $P E R_{1}<P E R_{2}$, the solution to the dual-regime queue is unique in $\alpha$ when $x^{\star}=$ $B$.

Proof 2. Note that $G(B, \alpha)$ is monotonically increasing in $\alpha$ at $x=B$. Let $\left(B, \alpha^{\star}\right)$ be a solution, then for any $\alpha^{\prime} \neq$ $\alpha^{\star}, G\left(B, \alpha^{\prime}\right) \neq G\left(B, \alpha^{\star}\right)=1-q(B)$, which concludes the proof.

We also outline an algorithm to numerically solve the dual-regime queue in Algorithm 1. Once the solution is assured to reside in either of the two regimes $\mathcal{R}_{1}$ or $\mathcal{R}_{2}$, or at the boundary $\mathcal{B}$, then a binary search is performed for the unknowns $x^{\star}$ and $\alpha^{\star}$, respectively, in the corresponding domain. We note that the case of $r_{1}=r_{2}$ and $P E R_{1}=P E R_{2}$ can also be solved by Algorithm 1 .

\section{Wireless link and traffic scenarios}

In our numerical examples, we use the following parameters. For the fixed packet size, we set $L=1500$ bytes. For GRED, the parameters $t h_{\min }$ and $t h_{\max }$ are set to 30 and 90, respectively, in units of packets, and $p_{\max }$ is set to 0.1 as in [43]. For TCP parameters, we set $T_{0, \min }=1 \mathrm{~s}$ as in [49], $b=2$ and $W=64$ kbytes as in [48]. We use the MCSs of the Wireless-MAN OFDMA PHY which specifies a cellular communication system comprising a base station (BS) and a number of mobile stations (MSs) [50]. The Wireless-MAN OFDMA PHY can alternatively be used as a PTP link as in the references [51] and [52]. The scope of this paper suits well to such PTP systems relying on OFDM-based air interfaces [53-55]. For Wireless-MAN OFDMA $P H Y$, we carry out simulations with the Coded Modulation Library (CML) to obtain the $\operatorname{per}_{m, s}$ values for given $\operatorname{mcs}_{m}$ and $s n r_{s}$ [56]. For this purpose, we choose eight MCSs that use convolutional turbo codes (CTC) which are enumerated in Table 1 according to an MCS index $m, m \in\{0,1, \ldots, 7\}$, for use in the current paper which differ according to their modulation order $V_{m}$ (i.e., the number of points in the constellation diagram), code rate $R_{m}$, and forward error correction (FEC) block length $k_{m}$.

Assuming FEC block error events of a packet to be iid Bernoulli distributed, the quantity per $_{m, s}$ can be derived from the FEC block error rate (FER) denoted by $\mathrm{fer}_{m, s}$ as follows:

$$
\operatorname{per}_{m, s}=1-\left(1-f e r_{m, s}\right)^{\left\lceil L / k_{m}\right\rceil} .
$$

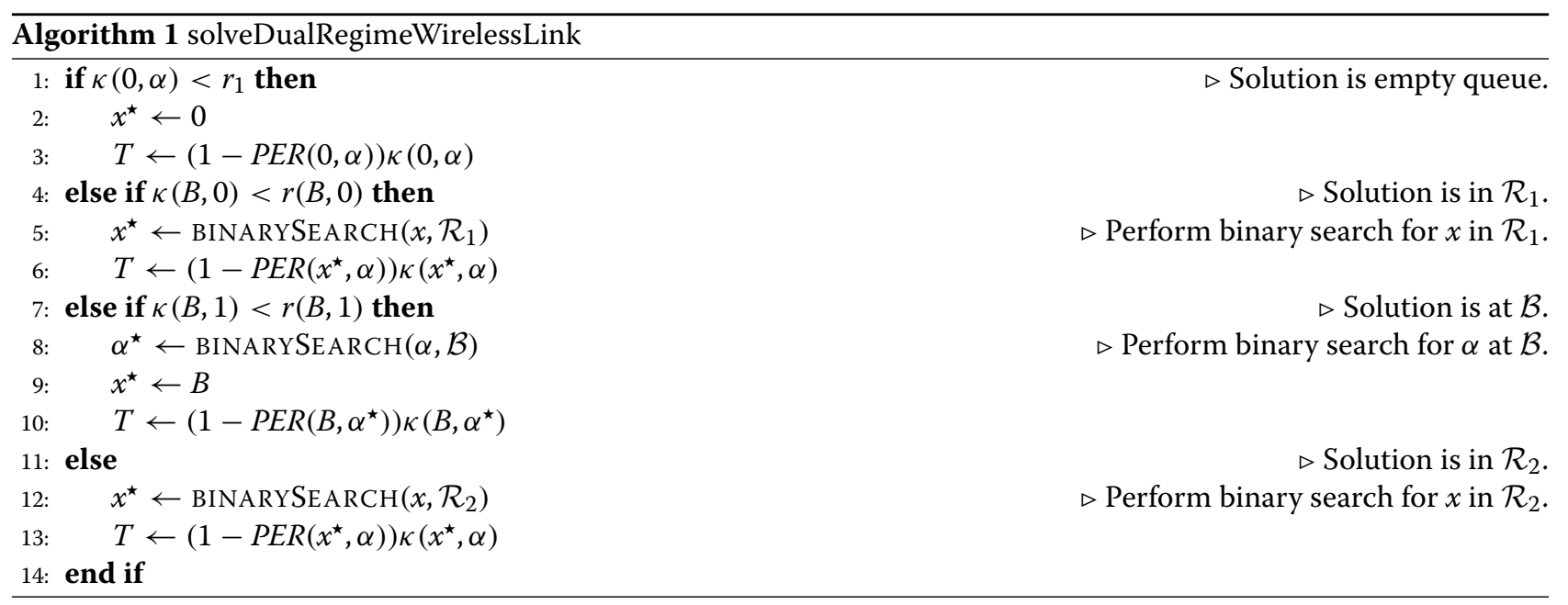


Table 1 Modulation and coding schemes of IEEE 802.16 used in the numerical examples

\begin{tabular}{lllllllll}
\hline$m$ & 0 & 1 & 2 & 3 & 4 & 5 & 6 & 7 \\
\hline$V_{m}$ & 4 & 4 & 16 & 16 & 64 & 64 & 64 & 64 \\
$R_{m}$ & $1 / 2$ & $3 / 4$ & $1 / 2$ & $3 / 4$ & $1 / 2$ & $2 / 3$ & $3 / 4$ & $5 / 6$ \\
$k_{m}$ (bytes) & 60 & 54 & 60 & 54 & 54 & 48 & 54 & 60 \\
\hline
\end{tabular}

For the sake of completeness, we present per $_{m, s}$ vs. $s n r_{s}$ curves obtained using CML in Figs. 2 and 3, for the additive white Gaussian noise (AWGN) and the ITU vehicular-A channels, respectively, the latter corresponding to an MS with velocity $90 \mathrm{~km} / \mathrm{h}$, which is referred to as the ITU-A channel for the rest of the paper [57]. SNR ranges of $[0 \mathrm{~dB}, 22 \mathrm{~dB}]$ and $[0 \mathrm{~dB}, 40 \mathrm{~dB}]$ are sampled with a resolution of 0.5 and $2 \mathrm{~dB}$, respectively, to obtain the corresponding $s n r_{s}$ values for the AWGN and the ITU-A channels. At least $10^{7} \mathrm{FEC}$ blocks are decoded to reach the PERs illustrated in these figures.

The time division duplex (TDD) mode as specified by WiMAX [58] uses 35 downlink (DL) OFDM symbols with 768 data sub-carriers per symbol for a channel bandwidth of $10 \mathrm{MHz}$ [50] and a TDD frame duration of 5 $\mathrm{ms}$, resulting in an average PHY rate of $c=5.37610^{6}$ sub-carriers/s. The raw bit rate $c_{m}$ of the IEEE 802.16 Wireless-MAN OFDMA PHY air interface can then be calculated in bps as $c_{m}=c \log _{2}\left(V_{m}\right) R_{m}$. The padding inefficiency caused by the need for FEC block alignment of packets reduces the raw bit rate down to the link layer bit rate $g_{m}=c_{m} L /\left(k_{m}\left\lceil L / k_{m}\right\rceil\right)$ for MCS $m c s_{m}$. Note that
MCSs in Table 1 are ordered based on their raw bit rates. These eight different MCSs lead to $\frac{8 * 7}{2}-1=27$ possible DRWL policies which satisfy the condition $r_{1}<r_{2}$ and $P E R_{1}<P E R_{2}$, excluding in particular the dual-regime policy $\left(M C S_{1}, M C S_{2}\right)=\left(m c s_{3}, m c s_{4}\right)$ having the same link layer bit rate (i.e., $g_{3}=g_{4}$ ) but with interchanging PER performances for the AWGN and the ITU-A channels. Taking into consideration the remaining policies for which $r_{1}=r_{2}$ and $P E R_{1}=P E R_{2}$, an overall of $27+8=35$ distinct policies are studied in the numerical examples. In order to account for framing and processing delays of the system, $D_{F}$ is set to $2.5 \mathrm{~ms}$.

We construct traffic scenarios spanning a wide range of $N$ and $R T T_{0, i}$ values. In particular, we study two groups of traffic scenarios having fixed and uniformly spaced $R T T_{0, i}$ values denoted by $S F_{N, F}$ and $S U_{N, F}$, respectively, where $F$ denotes the average value of $R T T_{0, i}$ of the corresponding scenario. In scenario $S F_{N, F}$, there are $N$ longlived TCP flows, and all flows have the same $R T T_{0, i}$ of $F$ ms. On the other hand, in scenario $S U_{N, F}$, there are again $N$ flows but each with a different $R T T_{0, i}=2(i+$ $1) F /(N+1)$ leading to an average fixed RTT of $F \mathrm{~ms}$. Wired RTTs of TCP connections in a $3 G$ network is reported to vary from a few milliseconds to a few hundreds of milliseconds [59]. In line with this observation, we vary $F$ from $1 \mathrm{~ms}$ to $100 \mathrm{~ms}$ particularly choosing $F \in\{1,5,10,20,40,60,80,100\} \mathrm{ms}$. Because of the distribution of individual $R T T_{0, i}$ values for the scenario group $S U_{N, F}$, the resulting actual RTT values cover a wider range than the interval between the minimum and maximum values chosen for $F$. We set the maximum value of $N$

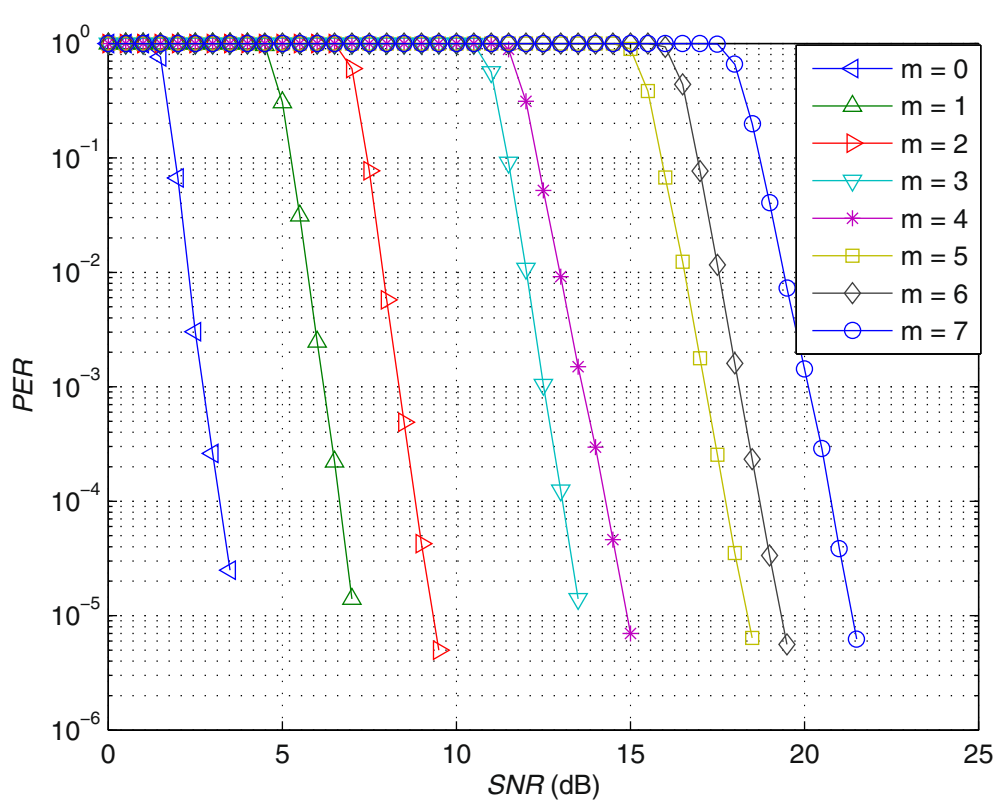

Fig. 2 Simulated PER per ${ }_{m, s}$ for different values of the MCS index $m$ and the AWGN channel 


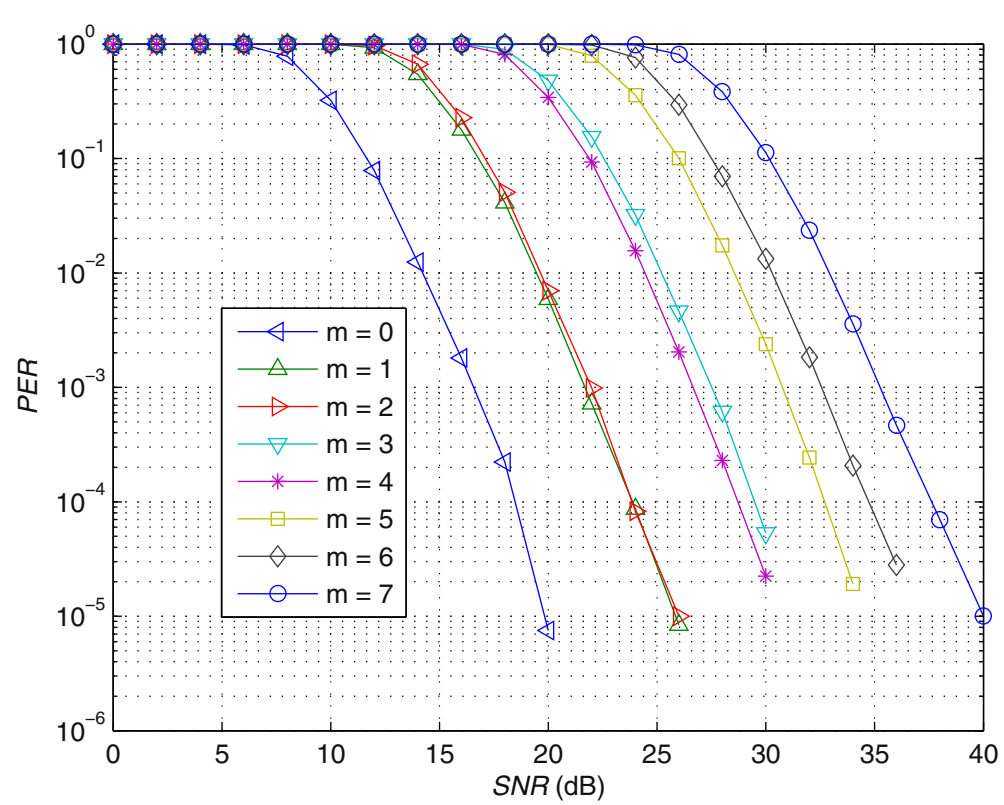

Fig. 3 Simulated PER per $m, s$ for different values of the MCS index $m$ and the ITU-A channel

to 16 as in the reference [60] for the wireless backbone topology studied therein and let $N \in\{1,2,4,8,16\}$. Note that scenarios represented by $S F_{1, F}$ and $S U_{1, F}$ are identical, thus leading to an overall of 72 unique traffic scenarios.

\section{Validation of the analytical model}

The proposed fixed-point analytical model for DRWL is validated using the ns-3 network simulator [48] for a subset of scenarios described in Section 4. Particularly, we employ the scenarios $S U_{N, F}$ for $N \in\{1,4,16\}, F \in\{1,40\}$ $\mathrm{ms}$, and $s n r_{s} \in\{22,26,30\} \mathrm{dB}$ for the ITU-A channel, totaling 18 distinct scenarios indexed by the parameter $i d x$. Table 2 summarizes the parameters used for each scenario including the per-regime PER values $\left(P E R_{1}, P E R_{2}\right)$, and per-regime bit rates $\left(r_{1}, r_{2}\right)$. We prefer to use a dumb-bell topology, which is a common topology to study TCP congestion algorithms in bottleneck links, involving $N$ TCP-Reno flows in our simulations as shown in Fig. $4[61,62]$. The ingress link for flow $i, 0 \leq i<$ $N$, has a one-way propagation delay $D_{R_{i}}$ whereas the egress link for the same flow has a one-way propagation delay $D_{L}$. We set $D_{L}=\min _{i}\left(R T T_{0, i}\right) / 4$ and $D_{R_{i}}=$ $R T T_{0, i} / 2-D_{L}$. All ingress and egress links have a capacity of 1 Gbps leaving the central wireless link in the middle as the bottleneck link with one-way propagation delay $D_{F}=2.5 \mathrm{~ms}$. Note that the central link is of DRWL-type with its MCS selection policy dictated by Table 2.

TCP flow statistics are obtained using the FlowMonitor which is a monitoring framework developed for ns-3
[63]. The RateErrorModel class of ns-3 is used to simulate PERs. Simulations are terminated after $5 \mathrm{~min}$, but the first $30 \mathrm{~s}$ corresponding to transients is ignored. Each simulation is repeated ten times and the average results are reported together with the $99 \%$ confidence intervals. Aggregate TCP throughput results of ns- 3 simulations and the analytical model proposed in this paper are presented for each scenario in Tables 3,4 , and 5 for $B$ being equal to 10,20 , and 30 , respectively, all in units of packets. In these tables, we also provide the regime of the solution point (i.e., whether $x^{\star}$ resides in $\mathcal{R}_{1}, \mathcal{R}_{2}$, or at the boundary $\mathcal{B}$ ) and the $\alpha^{\star}$ parameter, whenever the solution is at $\mathcal{B}$, obtained by the analysis. Overall results exhibit a remarkable level of agreement in the aggregate TCP throughput between simulations and the fixed-point analytical model especially for $B$ equals 20 or 30 . For $B=$ 10 , which is closer to the vicinity of the empty queue, the analytical model tends to be optimistic for scenarios with high PER values for $\mathcal{R}_{2}$ (e.g., $i d x$ equals 2,11 , 12 , and 17). In order to better understand this behavior, we first let the discrete random variable $K$ denote the number of packets waiting in the queue for transmission with the probability mass function (PMF) $u_{k}$ defined as $u_{k}=\operatorname{Pr}(K=k)$ where $k \in\left[0,2 t h_{\max }\right)$. Subsequently, we present the empirical PMF $u_{k}$ obtained from ns-3 simulations for three different regime boundary values when the scenario index is fixed at $i d x=2$ in Fig. 5. Note that in ns-3, which is an event-based simulator, it is more convenient to probe the queue occupancy in units of packets rather than the continuous occupancy level $x$. The analytical queue occupancy, however, is a real number which 
Table 2 The list of 18 traffic scenarios indexed with idx used for validation of the fixed-point analytical model proposed for DRWL for the ITU-A channel model

\begin{tabular}{|c|c|c|c|c|c|c|c|c|}
\hline$i d x$ & Traffic scenario & SNR (dB) & $M C S_{1}$ & $M C S_{2}$ & $r_{1}$ (Mbps) & $r_{2}$ (Mbps) & $P E R_{1}$ & $P E R_{2}$ \\
\hline 1 & $S U_{1,1}$ & 22 & $m c s_{1}$ & $\mathrm{mcs}_{3}$ & 8 & 16 & $7.1910^{-4}$ & $1.5510^{-1}$ \\
\hline 2 & $S U_{1,1}$ & 26 & $\mathrm{mcs}_{4}$ & $\operatorname{mcs}_{5}$ & 16 & 21 & $2.0410^{-3}$ & $1.0010^{-1}$ \\
\hline 3 & $S U_{1,1}$ & 30 & $\mathrm{mcs}_{4}$ & $\operatorname{mcs}_{5}$ & 16 & 21 & $2.2410^{-5}$ & $2.3910^{-3}$ \\
\hline 4 & $S U_{1,40}$ & 22 & $m c s_{0}$ & $m c s_{1}$ & 5.376 & 8 & 0 & $7.1910^{-4}$ \\
\hline 5 & $S U_{1,40}$ & 26 & mcso & $m c s_{1}$ & 5.376 & 8 & 0 & $8.4010^{-6}$ \\
\hline 6 & $S U_{1,40}$ & 30 & mcso & $m c_{7}$ & 5.376 & 26.88 & 0 & $1.1210^{-1}$ \\
\hline 7 & $S U_{4,1}$ & 22 & $m c s_{1}$ & $m \mathrm{~ms}_{2}$ & 8 & 10.752 & $7.1910^{-4}$ & $9.8510^{-4}$ \\
\hline 8 & $S U_{4,1}$ & 26 & $\mathrm{mcs}_{2}$ & $\mathrm{mCs}_{5}$ & 10.752 & 21 & $1.0010^{-5}$ & $1.0010^{-1}$ \\
\hline 9 & $S U_{4,1}$ & 30 & $\operatorname{mcs}_{5}$ & $m c_{7}$ & 21 & 26.88 & $2.3910^{-3}$ & $1.1210^{-1}$ \\
\hline 10 & $S U_{4,40}$ & 22 & $m c s_{1}$ & $m \mathrm{~ms}_{2}$ & 8 & 10.752 & $7.1910^{-4}$ & $9.8510^{-4}$ \\
\hline 11 & $S U_{4,40}$ & 26 & $\mathrm{mcs}_{3}$ & $m c s_{6}$ & 16 & 24 & $4.6210^{-3}$ & $2.9510^{-1}$ \\
\hline 12 & $S U_{4,40}$ & 30 & $\mathrm{mcs}_{3}$ & $m c_{7}$ & 16 & 26.88 & $5.3210^{-5}$ & $1.1210^{-1}$ \\
\hline 13 & $S U_{16,1}$ & 22 & $\mathrm{mcs}_{2}$ & $\mathrm{mcs}_{4}$ & 10.752 & 16 & $9.8510^{-4}$ & $9.3210^{-2}$ \\
\hline 14 & $S U_{16,1}$ & 26 & $\mathrm{mcs}_{2}$ & $m c s_{6}$ & 10.752 & 24 & $1.0010^{-5}$ & $2.9510^{-1}$ \\
\hline 15 & $S U_{16,1}$ & 30 & $\mathrm{mcs}_{2}$ & $m c s_{6}$ & 10.752 & 24 & 0 & $1.3310^{-2}$ \\
\hline 16 & $S U_{16,40}$ & 22 & $m c s_{1}$ & $m c s_{4}$ & 8 & 16 & $7.1910^{-4}$ & $9.3210^{-2}$ \\
\hline 17 & $S U_{16,40}$ & 26 & $\mathrm{mcs}_{3}$ & $\operatorname{mcs}_{5}$ & 16 & 21 & $4.6210^{-3}$ & $1.0010^{-1}$ \\
\hline 18 & $S U_{16,40}$ & 30 & $\mathrm{mCs}_{3}$ & $\mathrm{mcs}_{5}$ & 16 & 21 & $5.3210^{-5}$ & $2.3910^{-3}$ \\
\hline
\end{tabular}

is found to be 3.9049 in units of packets for all values of $B$. By its very nature, the fixed-point approach makes the location of $\mathcal{B}$ irrelevant to the solution once the solution resides in either $\mathcal{R}_{1}$ or $\mathcal{R}_{2}$. Accordingly, for decreasing $B$, the probability of queue becoming empty obtained from simulations increases which is not accounted by the model for this particular case.

In Fig. 6, the empirical queue occupancy PMF obtained with ns-3 simulations is depicted together with the corresponding analytical solution for $B=20$ corresponding to 6 scenarios with $i d x \in\{3,4,11,13,14,18\}$. When idx equals 11 and 18, the model finds the solution in $\mathcal{R}_{1}$ and $\mathcal{R}_{2}$, respectively, and for $i d x$ equals $3,4,13$, or 14 , the solution turns out to be at $\mathcal{B}$. The shape of the PMFs obtained via ns-3 for scenarios with analytical solution at $\mathcal{B}$ demonstrates the effectiveness of the proposed approach in capturing the behavior of the queue around the regime boundary. In the light of all simulation results, we let $B \geq 20$ in all the remaining numerical examples so that the empty queue regime is avoided and furthermore the fixed-point model performs remarkably well.

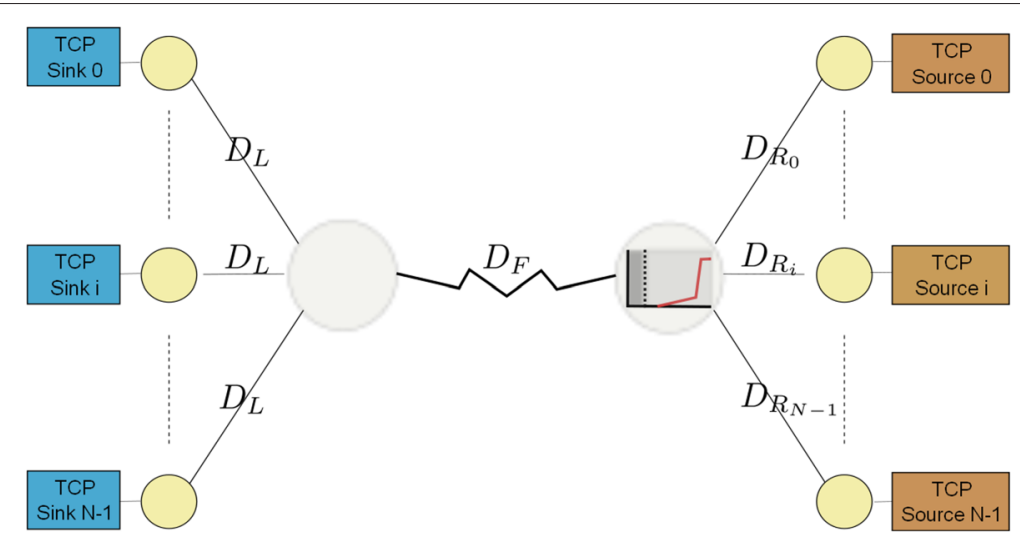

Fig. 4 ns-3 simulation topology 
Table 3 Aggregate TCP throughput $T$ obtained with ns-3 simulations and the fixed-point analytical model for $B=10$. Results for ns-3 simulations are presented with the $99 \%$ confidence intervals

\begin{tabular}{|c|c|c|c|c|c|}
\hline$i d x$ & $\begin{array}{l}T \text { (ns3) } \\
\text { (Mbps) }\end{array}$ & $\begin{array}{l}T \text { (analysis) } \\
\text { (Mbps) }\end{array}$ & $\begin{array}{l}\text { Solution } \\
\text { domain }\end{array}$ & $\begin{array}{l}x^{\star} \\
\text { (packets) }\end{array}$ & $\alpha^{\star}$ \\
\hline 1 & $7.7536 \pm 0.0535$ & 8.0208 & $\mathcal{B}$ & 10.0000 & $4.8110^{-3}$ \\
\hline 2 & $13.2888 \pm 0.4525$ & 15.9673 & $\mathcal{R}_{1}$ & 3.9049 & $\mathrm{~N} / \mathrm{A}$ \\
\hline 3 & $18.5577 \pm 0.0686$ & 18.2195 & $\mathcal{B}$ & 10.0000 & $4.4810^{-1}$ \\
\hline 4 & $6.5931 \pm 0.0939$ & 6.5793 & $\mathcal{B}$ & 10.0000 & $4.6010^{-1}$ \\
\hline 5 & $7.9630 \pm 0.0169$ & 7.9999 & $\mathcal{R}_{2}$ & 12.4837 & $\mathrm{~N} / \mathrm{A}$ \\
\hline 6 & $4.9082 \pm 0.0531$ & 5.3999 & $\mathcal{B}$ & 10.0000 & $1.2910^{-3}$ \\
\hline 7 & $10.7244 \pm 0.0013$ & 10.7414 & $\mathcal{R}_{2}$ & 32.3563 & $\mathrm{~N} / \mathrm{A}$ \\
\hline 8 & $11.3006 \pm 0.0171$ & 11.1503 & $\mathcal{B}$ & 10.0000 & $4.9010^{-2}$ \\
\hline 9 & $20.1596 \pm 0.0843$ & 21.0230 & $\mathcal{B}$ & 10.0000 & $2.5110^{-2}$ \\
\hline 10 & $10.6419 \pm 0.0119$ & 10.7412 & $\mathcal{R}_{2}$ & 30.7266 & N/A \\
\hline 11 & $10.6183 \pm 0.1630$ & 12.0195 & $\mathcal{R}_{1}$ & 0.0000 & N/A \\
\hline 12 & $14.2814 \pm 0.1153$ & 16.0956 & $\mathcal{B}$ & 10.0000 & $1.2310^{-2}$ \\
\hline 13 & $11.6557 \pm 0.0193$ & 11.6082 & $\mathcal{B}$ & 10.0000 & $2.3010^{-1}$ \\
\hline 14 & $11.0416 \pm 0.0092$ & 11.0528 & $\mathcal{B}$ & 10.0000 & $4.8810^{-2}$ \\
\hline 15 & $23.5724 \pm 0.0224$ & 23.6809 & $\mathcal{R}_{2}$ & 31.1479 & $\mathrm{~N} / \mathrm{A}$ \\
\hline 16 & $8.9470 \pm 0.0216$ & 9.0075 & $\mathcal{B}$ & 10.0000 & $1.5610^{-1}$ \\
\hline 17 & $14.4627 \pm 0.0720$ & 16.1824 & $\mathcal{B}$ & 10.0000 & $8.6510^{-2}$ \\
\hline 18 & $20.8878 \pm 0.0041$ & 20.9496 & $\mathcal{R}_{2}$ & 34.1783 & N/A \\
\hline
\end{tabular}

Table 4 Aggregate TCP throughput T obtained with ns-3 simulations and the fixed-point analytical model for $B=20$. Results for ns-3 simulations are presented with the $99 \%$ confidence intervals

\begin{tabular}{|c|c|c|c|c|c|}
\hline$i d x$ & $\begin{array}{l}T \text { (ns-3) } \\
\text { (Mbps) }\end{array}$ & $\begin{array}{l}T \text { (analysis) } \\
\text { (Mbps) }\end{array}$ & $\begin{array}{l}\text { Solution } \\
\text { domain }\end{array}$ & $\begin{array}{l}x^{\star} \\
\text { (packets) }\end{array}$ & $\alpha^{\star}$ \\
\hline 1 & $7.9566 \pm 0.0315$ & 8.0001 & $\mathcal{B}$ & 20.0000 & $1.0610^{-3}$ \\
\hline 2 & $15.0448 \pm 0.2067$ & 15.9673 & $\mathcal{R}_{1}$ & 3.9049 & N/A \\
\hline 3 & $17.2953 \pm 0.1523$ & 17.2050 & $\mathcal{B}$ & 20.0000 & $2.4410^{-1}$ \\
\hline 4 & $5.8687 \pm 0.0183$ & 5.5724 & $\mathcal{B}$ & 20.0000 & $7.5010^{-2}$ \\
\hline 5 & $6.1174 \pm 0.0069$ & 6.0328 & $\mathcal{B}$ & 20.0000 & $2.5010^{-1}$ \\
\hline 6 & $4.9370 \pm 0.0696$ & 5.3797 & $\mathcal{B}$ & 20.0000 & $1.9810^{-4}$ \\
\hline 7 & $10.6713 \pm 0.0099$ & 10.7414 & $\mathcal{R}_{2}$ & 32.3564 & N/A \\
\hline 8 & $11.1897 \pm 0.0077$ & 11.0506 & $\mathcal{B}$ & 20.0000 & $3.6710^{-2}$ \\
\hline 9 & $20.9866 \pm 0.0253$ & 20.9979 & $\mathcal{B}$ & 20.0000 & $1.6510^{-2}$ \\
\hline 10 & $10.2652 \pm 0.0249$ & 10.7412 & $\mathcal{R}_{2}$ & 30.7266 & N/A \\
\hline 11 & $12.7334 \pm 0.1325$ & 12.0195 & $\mathcal{R}_{1}$ & 0.0000 & N/A \\
\hline 12 & $15.6795 \pm 0.0350$ & 16.0741 & $\mathcal{B}$ & 20.0000 & $9.5310^{-3}$ \\
\hline 13 & $11.5683 \pm 0.0102$ & 11.5174 & $\mathcal{B}$ & 20.0000 & $2.0610^{-1}$ \\
\hline 14 & $11.0345 \pm 0.0066$ & 11.0222 & $\mathcal{B}$ & 20.0000 & $4.3910^{-2}$ \\
\hline 15 & $23.1019 \pm 0.0520$ & 23.6811 & $\mathcal{R}_{2}$ & 31.1479 & N/A \\
\hline 16 & $8.9518 \pm 0.0165$ & 8.8827 & $\mathcal{B}$ & 20.0000 & $1.3610^{-1}$ \\
\hline 17 & $15.9371 \pm 0.0321$ & 16.1458 & $\mathcal{B}$ & 20.0000 & $7.4110^{-2}$ \\
\hline 18 & $20.6509 \pm 0.0187$ & 20.9499 & $\mathcal{R}_{2}$ & 34.1782 & N/A \\
\hline
\end{tabular}


Table 5 Aggregate TCP throughput $T$ obtained with ns-3 simulations and the fixed-point analytical model for $B=30$. Results for ns-3 simulations are presented with the $99 \%$ confidence intervals

\begin{tabular}{|c|c|c|c|c|c|}
\hline$i d x$ & $\begin{array}{l}T \text { (ns3) } \\
\text { (Mbps) }\end{array}$ & $\begin{array}{l}T \text { (analysis) } \\
\text { (Mbps) }\end{array}$ & $\begin{array}{l}\text { Solution } \\
\text { domain }\end{array}$ & $\begin{array}{l}x^{\star} \\
\text { (packets) }\end{array}$ & $\alpha^{\star}$ \\
\hline 1 & $7.9785 \pm 0.0162$ & 7.9942 & $\mathcal{R}_{1}$ & 25.9733 & N/A \\
\hline 2 & $15.4886 \pm 0.0857$ & 15.9674 & $\mathcal{R}_{1}$ & 3.9049 & N/A \\
\hline 3 & $15.9636 \pm 0.2527$ & 16.2673 & $\mathcal{B}$ & 30.0000 & $5.4110^{-2}$ \\
\hline 4 & $5.3673 \pm 0.0000$ & 5.3760 & $\mathcal{R}_{1}$ & 22.5306 & $\mathrm{~N} / \mathrm{A}$ \\
\hline 5 & $5.3673 \pm 0.0000$ & 5.3760 & $\mathcal{R}_{1}$ & 22.5306 & N/A \\
\hline 6 & $5.3673 \pm 0.0000$ & 5.3760 & $\mathcal{R}_{1}$ & 22.5306 & N/A \\
\hline 7 & $9.8443 \pm 0.0336$ & 10.7416 & $\mathcal{R}_{2}$ & 32.3563 & $\mathrm{~N} / \mathrm{A}$ \\
\hline 8 & $11.0671 \pm 0.0080$ & 10.9739 & $\mathcal{B}$ & 30.0000 & $2.7310^{-2}$ \\
\hline 9 & $21.0011 \pm 0.0053$ & 20.9776 & $\mathcal{B}$ & 30.0000 & $9.5110^{-3}$ \\
\hline 10 & $9.1072 \pm 0.0330$ & 10.7412 & $\mathcal{R}_{2}$ & 30.7266 & N/A \\
\hline 11 & $12.9934 \pm 0.0867$ & 12.0195 & $\mathcal{R}_{1}$ & 0.0000 & $\mathrm{~N} / \mathrm{A}$ \\
\hline 12 & $15.9259 \pm 0.0378$ & 16.0586 & $\mathcal{B}$ & 30.0000 & $7.5610^{-3}$ \\
\hline 13 & $11.4676 \pm 0.0120$ & 11.4330 & $\mathcal{B}$ & 30.0000 & $1.8410^{-1}$ \\
\hline 14 & $11.0026 \pm 0.0043$ & 10.9937 & $\mathcal{B}$ & 30.0000 & $3.9310^{-2}$ \\
\hline 15 & $20.8078 \pm 0.1013$ & 23.6811 & $\mathcal{R}_{2}$ & 31.1479 & N/A \\
\hline 16 & $8.8136 \pm 0.0159$ & 8.7706 & $\mathcal{B}$ & 30.0000 & $1.1910^{-1}$ \\
\hline 17 & $16.1202 \pm 0.0134$ & 16.1126 & $\mathcal{B}$ & 30.0000 & $6.2910^{-2}$ \\
\hline 18 & $19.5952 \pm 0.0287$ & 20.9496 & $\mathcal{R}_{2}$ & 34.1783 & N/A \\
\hline
\end{tabular}

N/A not available

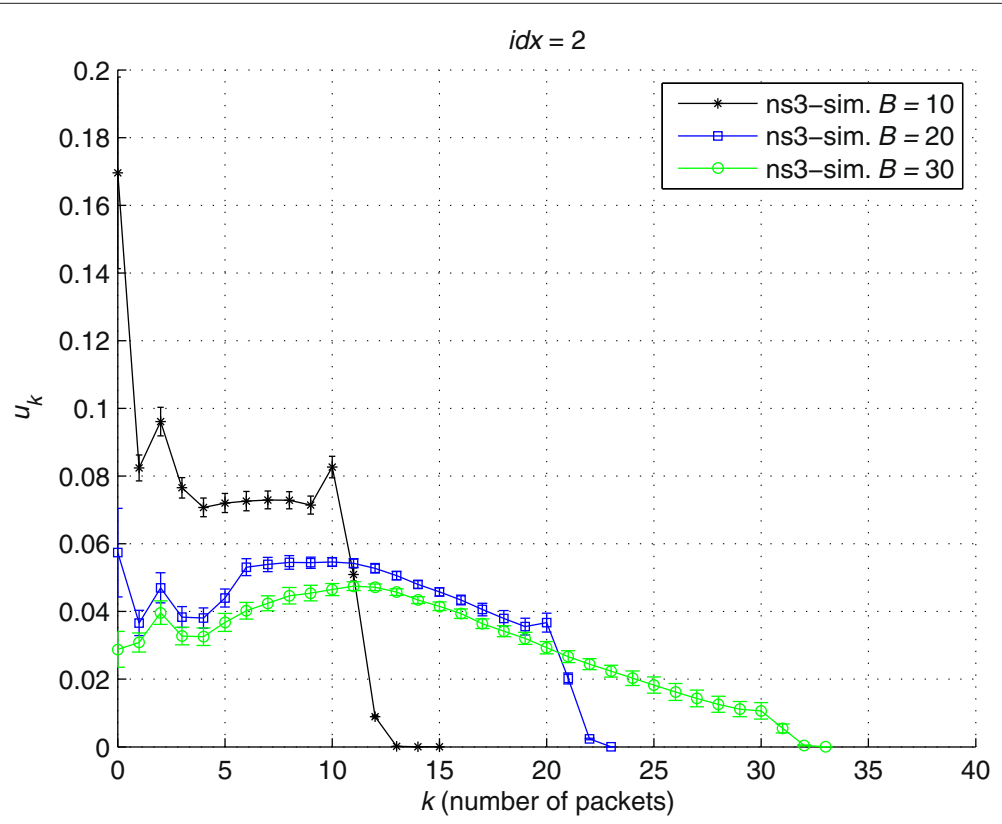

Fig. 5 The empirical queue occupancy PMF $u_{k}$ for the scenario with $i d x=2$ for varying $B \in\{10,20,30\}$, all having the same fixed-point solution with a queue occupancy level of 3.9049 packets 


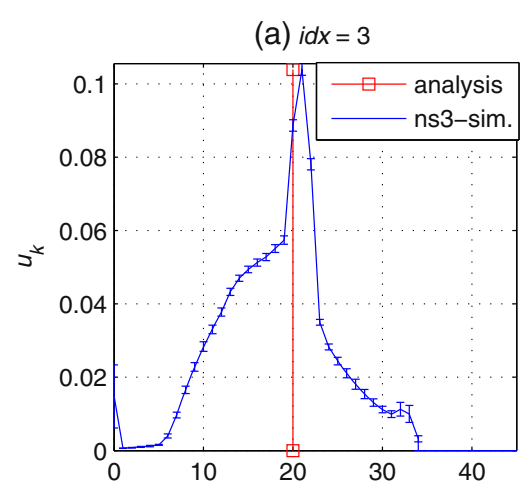

(c) $i d x=11$

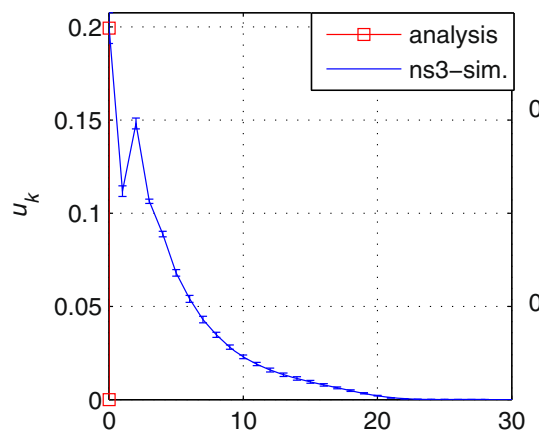

(e) $i d x=14$

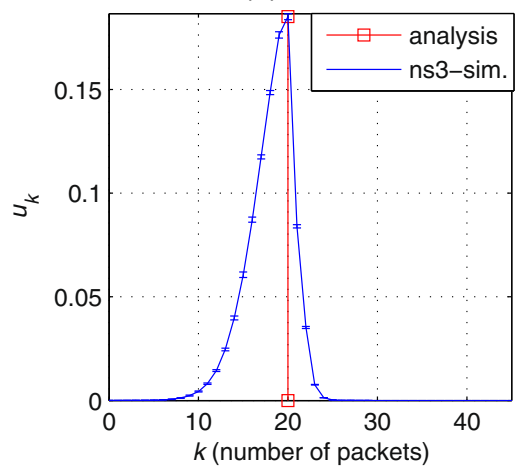

(b) $i d x=4$

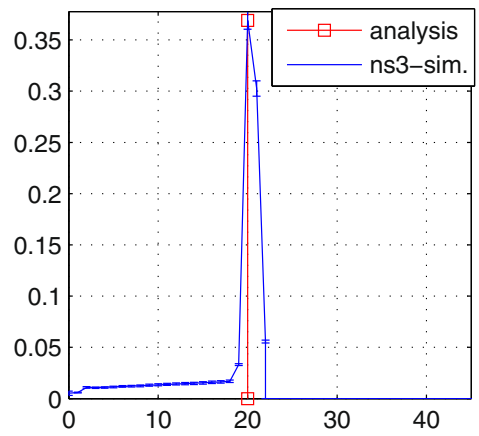

(d) $i d x=13$

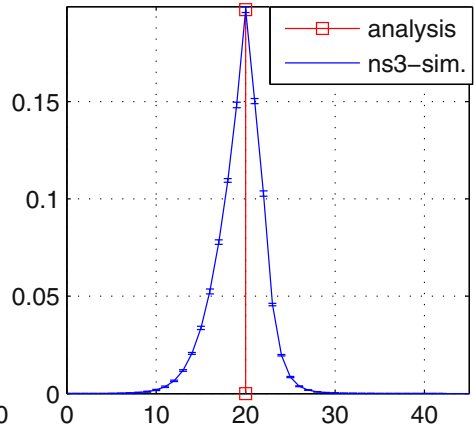

(f) $i d x=18$

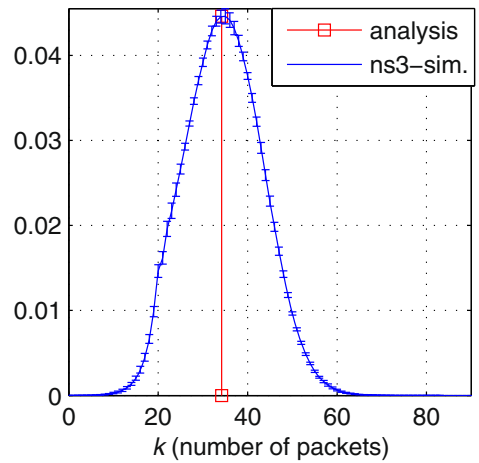

Fig. 6 The empirical queue occupancy PMF $u_{k}$ for scenarios with $B=20$ and $i d x \in\{3,4,11,13,14,18\}$ shown in panels (a-f), respectively. Analytical results are also depicted

\section{Performance evaluation of QAWLA}

For each $s n r_{s}$ and $t h_{P E R}$ value, we choose MCSs for both TAGLA and QAWLA policies. Out of all DRWL policies, we pick the one, called optimum, that produces the maximum TCP throughput for each $s n r_{s}$ value and traffic scenario, which is solely used for benchmarking due to the off-line nature of finding the optimum policy. For all figures to be presented, aggregate TCP throughput values of TAGLA and QAWLA are normalized with respect to the corresponding throughput values of optimum. In Figs. 7 and 8, normalized aggregate TCP throughput of TAGLA and QAWLA are averaged over all traffic scenarios and $s n r_{s}$ values to give the mean normalized aggregate TCP throughput $\boldsymbol{T}_{\boldsymbol{M}}$ for the AWGN and the
ITU-A channels, respectively, and plotted as a function of the threshold parameter $t_{P E R}$ for different values of $H$ and $B$ parameters of the QAWLA policy. Owing to shadow fading, the channel SNR is assumed to be a normal random variable with a mean of $12 \mathrm{~dB}(26 \mathrm{~dB})$ and a standard deviation of $8 \mathrm{~dB}$ for the AWGN (ITU-A) channel. We first discretize the normal distribution with a resolution of $0.5 \mathrm{~dB}(2 \mathrm{~dB})$ and then truncate over the support of [2 dB, $22 \mathrm{~dB}]([14 \mathrm{~dB}, 40 \mathrm{~dB}])$ for the AWGN (ITU-A) channel for averaging purposes over the $s n r_{s}$ values. The minimum SNR value for the support of each channel type is selected such that $m c s_{0}$ has a PER less than 0.1.

For both channel types, but more prominently for the ITU-A channel, the choice of $H=100$ yields the best 


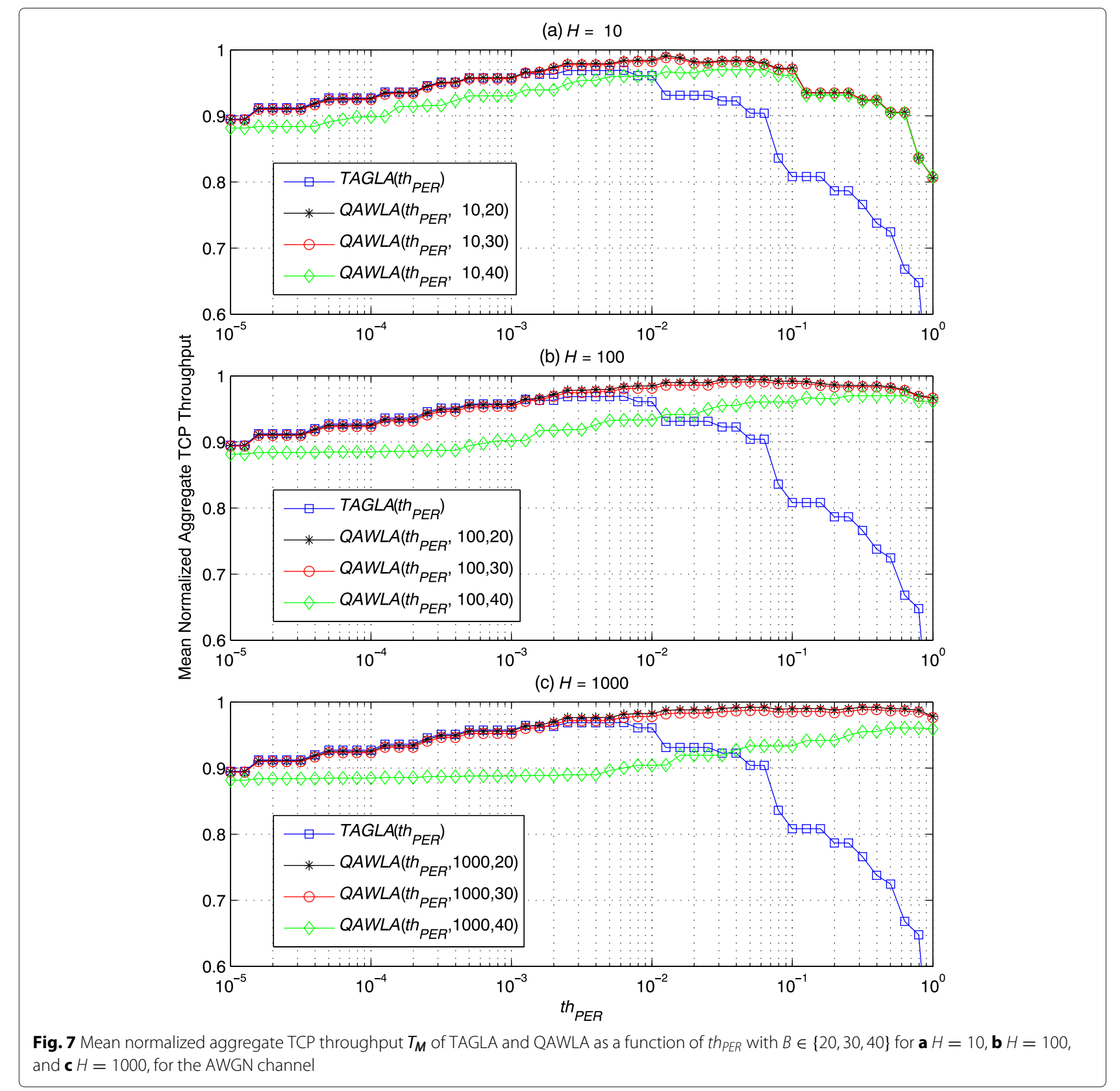

results in terms of peak normalized throughput and the degree of robustness for increasing $t h_{P E R}$ values. Note that QAWLA with $B=40>t h_{\min }=30$ performs worse than TAGLA for low values of $t h_{P E R}$, since AQM packet losses force the queue to be confined in $\mathcal{R}_{1}$ having lower capacity for the QAWLA scheme. Only for values of $t h_{P E R}$ well exceeding the level of $10^{-2}$ can the wireless packet losses start to dominate making $\mathcal{R}_{1}$ a desirable regime with its lower PER values for QAWLA. The remaining choices of $B \leq t h_{\min }$ reveal the true potential of QAWLA in both channels, while the $B=20$ choice performing slightly better than the choice of $B=30$ owing to its lower average queuing delay. For the rest of the numerical examples, we fix $H=100$ and $B=20$ and study the selection of the $t h_{P E R}$ parameter of the $Q A W L A\left(t_{P E R}, 100,20\right)$ policy.

In real-life implementations, both TAGLA and QAWLA policies require real-time PER estimations to successfully operate. This information, however, may not be precise due to rapidly changing wireless channel conditions and/or the accuracy of the particular implementations used in channel quality measurements. For example, the Wireless-MAN OFDMA PHY requires an absolute accuracy of $2 \mathrm{~dB}$ in the carrier-to-interference-and-noise ratio (CINR) measurements. In Figs. 9 and 10, throughput 


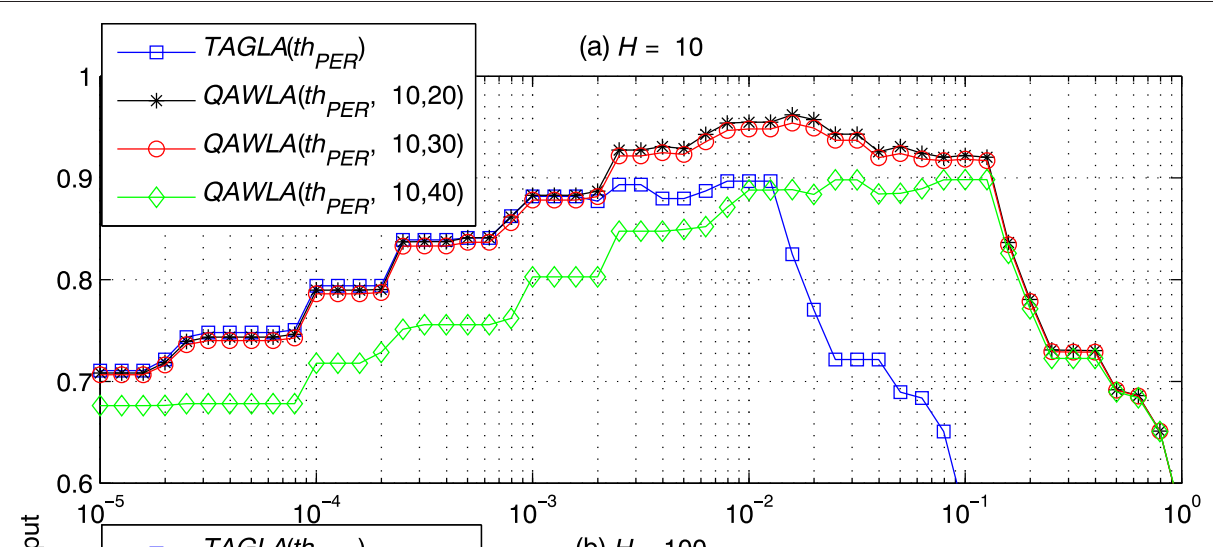

(b) $H=100$

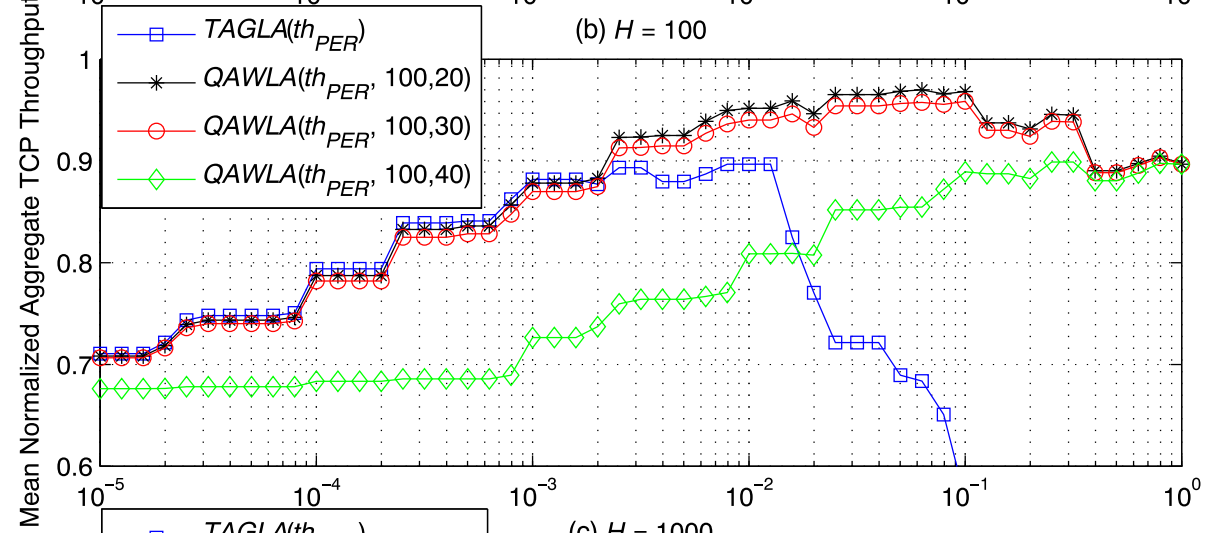

(c) $H=1000$

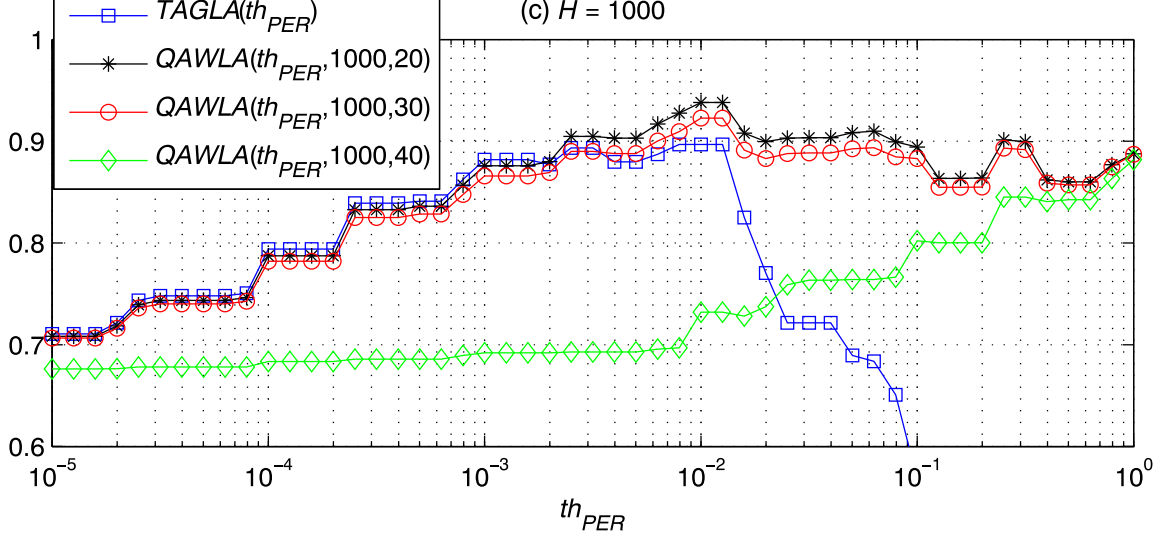

Fig. 8 Mean normalized aggregate TCP throughput $T_{M}$ of TAGLA and QAWLA as a function of th $_{\text {PER }}$ with $B \in\{20,30,40\}$ for $\mathbf{a} H=10, \mathbf{b} H=100$, and $\mathbf{c} H=1000$, for the ITU-A channel

results of TAGLA and QAWLA based on the MCS decisions given as if the channel SNR values were 2 and $4 \mathrm{~dB}$ higher than the actual values, resulting in an over-rated channel assessment, are also given. The same averaging steps described for Figs. 7 and 8 are employed for Figs. 9 and 10 in obtaining the presented throughput results. QAWLA appears to be relatively insensitive to the choice of $t h_{P E R}$ compared to TAGLA and significantly improves TAGLA for over-rated channels as well. Both TAGLA and QAWLA performances deteriorate in case an over-rated channel assessment is made, but the deterioration is more severe for the AWGN channel scenario as its $\mathrm{per}_{m, s}$ vs. $s n r_{s}$ curves are steeper than those of the ITU-A channel scenario. The optimum choice of the parameter $t h_{P E R}$ appears to be dependent on the channel type and the precision of the SNR estimate.

As an alternative metric, we define the worst case normalized aggregate TCP throughput $T_{W}$. In order to calculate $\boldsymbol{T}_{W}$, we first find the minimum value of the normalized aggregate TCP throughput for each traffic scenario over the aforementioned SNR values and then take average of these minimum values over the entire traffic scenario set. In Figs. 11 and 12, $\boldsymbol{T}_{\boldsymbol{W}}$ for TAGLA and QAWLA are depicted. The results clearly demonstrate 


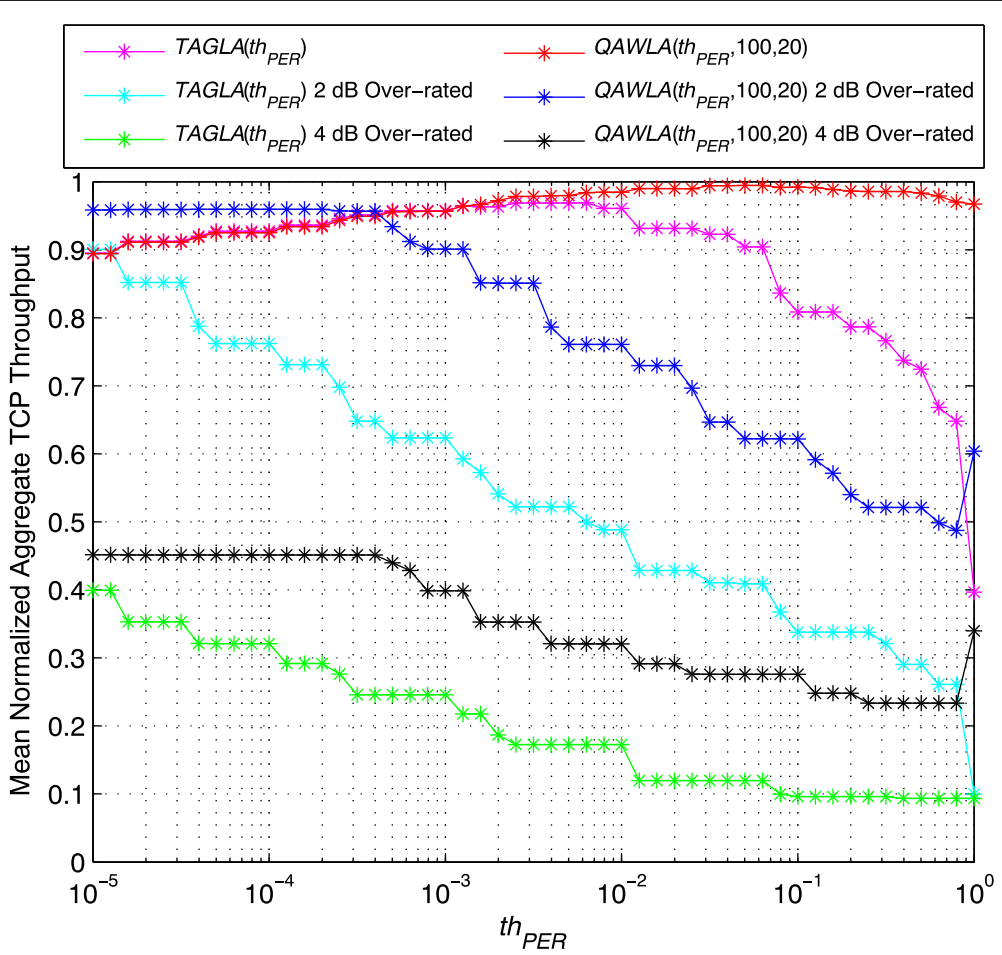

Fig. 9 Mean normalized aggregate TCP throughput $T_{M}$ of TAGLA and QAWLA as a function of th PER $_{\text {with }} H=100$ and $B=20$ for the AWGN channel

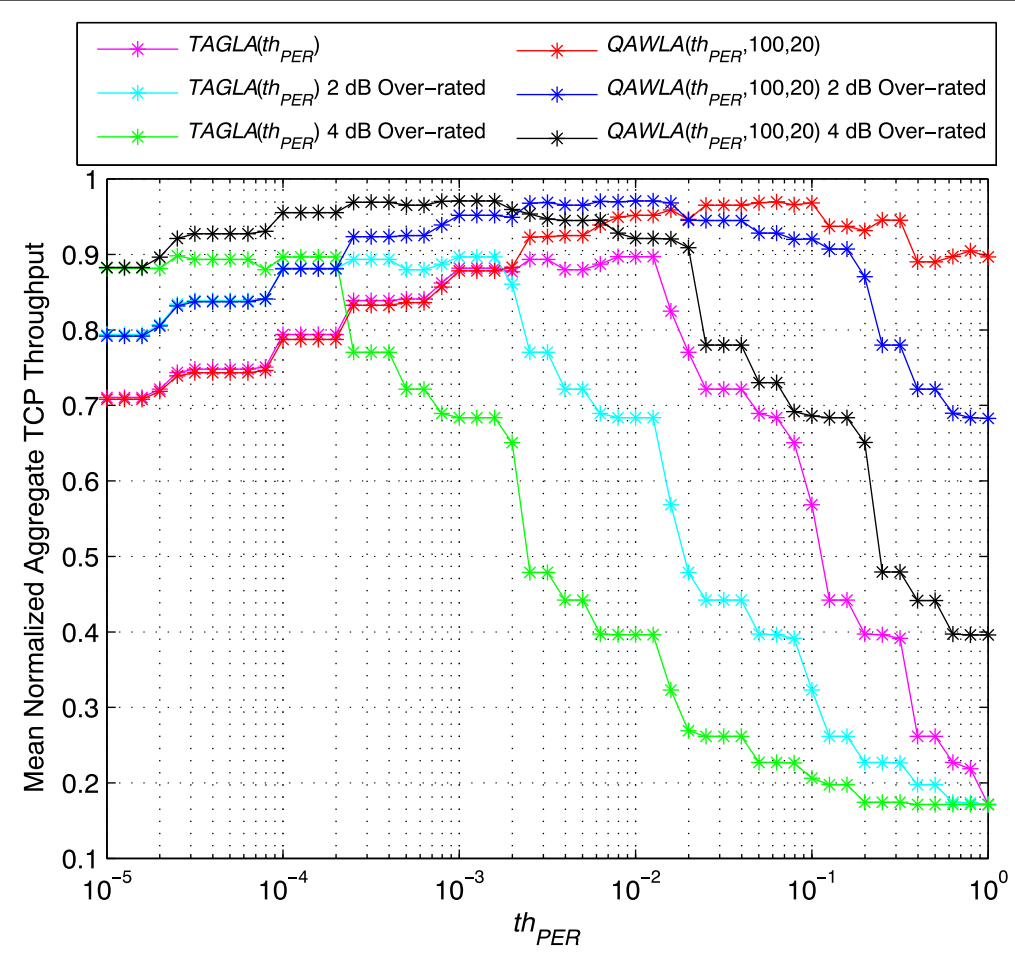

Fig. 10 Mean normalized aggregate TCP throughput $T_{M}$ of TAGLA and QAWLA as a function of thPER with $H=100$ and $B=20$ for the ITU-A channel 


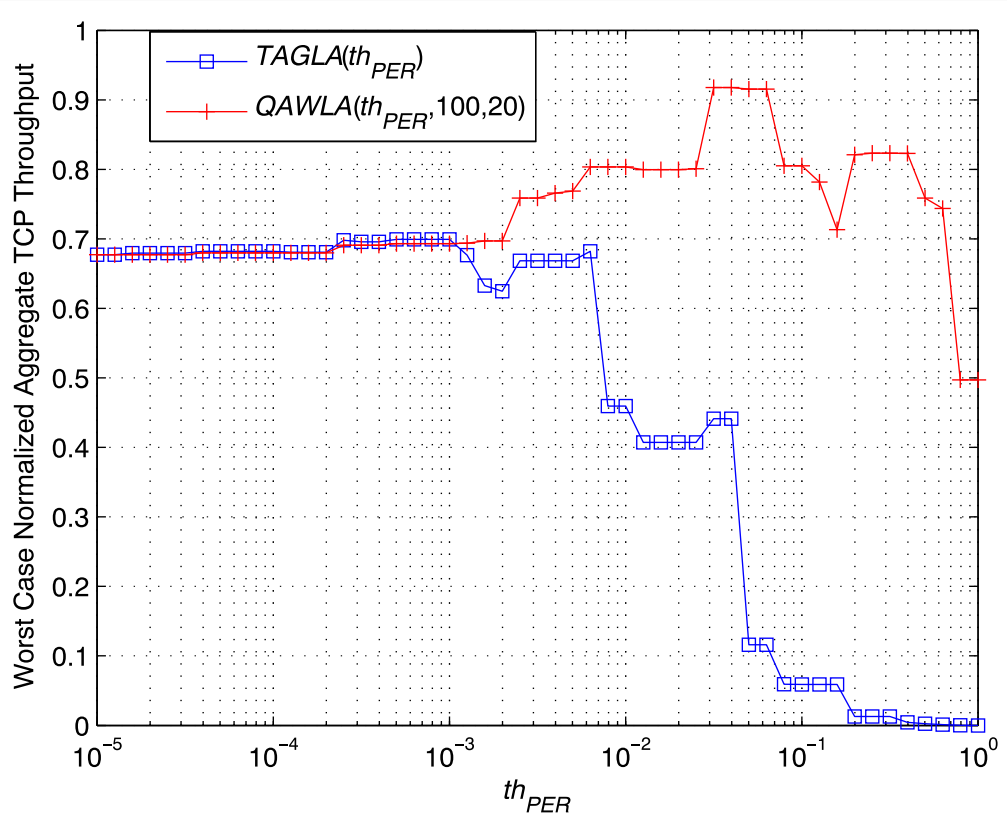

Fig. 11 Worst-case normalized aggregate TCP throughput $T_{W}$ of TAGLA and QAWLA as a function of th $h_{\text {EER }}$ with $H=100$ and $B=20$ for the AWGN channel

that the worst-case throughput performances of TAGLA are considerably improved with QAWLA. Unlike the overrated channel case, the worst-case throughput performances of both channels under TAGLA and QAWLA policies exhibit a similar trend. The worst-case performances $T_{W}$ of both channel types appear to peak in the vicinity of $t h_{P E R}=0.05$. This behavior is the consequence of the interplay between the bit rate and the packet loss rate of the chosen MCSs controlled by the parameter $t h_{P E R}$. In the light of the presented results, QAWLA proves to be superior to TAGLA. Moreover, the corresponding policy $Q A W L A(0.05,100,20)$ performs close to

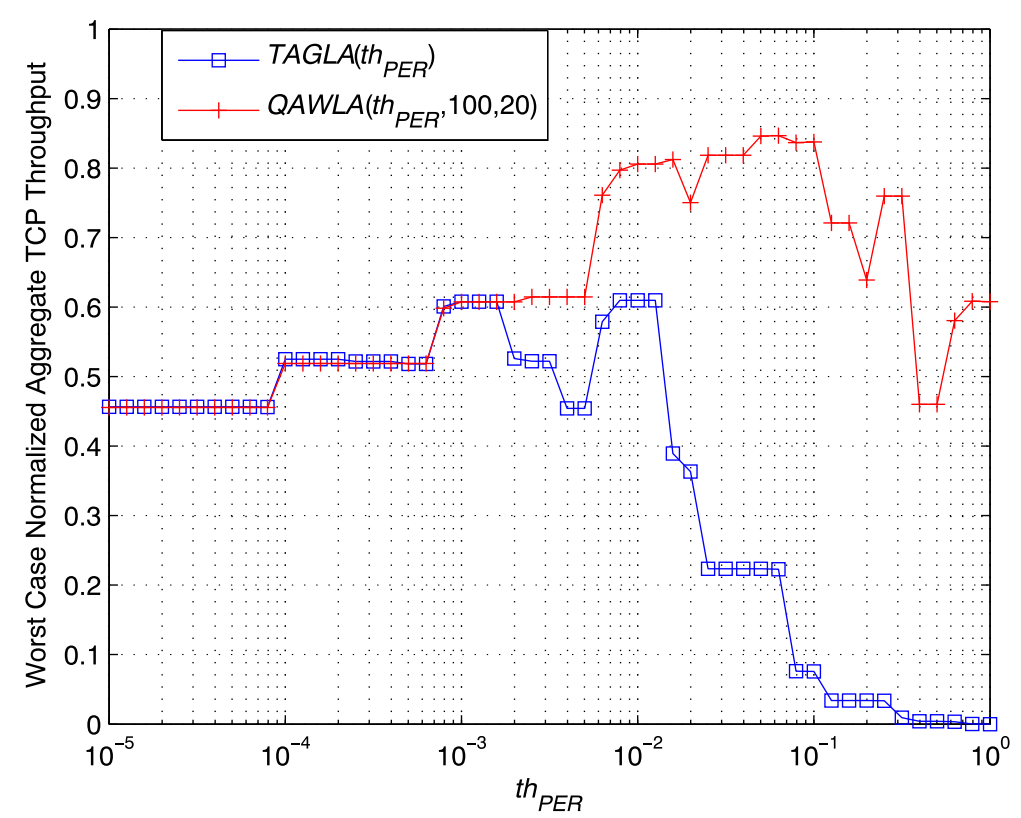

Fig. 12 Worst-case normalized aggregate TCP throughput $T_{W}$ of TAGLA and QAWLA as a function of thPER with $H=100$ and $B=20$ for the ITU-A channel 
optimum for both channel types as far as the mean performance $\boldsymbol{T}_{\boldsymbol{M}}$ is concerned and therefore appears to be a reasonable choice for the given AQM setting and the set of MCSs used in this study.

Finally, we compare QAWLA with other conventional LA schemes, namely target FER (TFER) and maximum PHY throughput (MaxPHYThru). TFER gives MCS decisions based solely and directly on the FER estimations as a function of its threshold parameter $t h_{F E R}$ and therefore denoted by TFER $\left(t h_{F E R}\right)[64,65]$. For a given SNR level $s n r_{s}$, TFER $\left(t h_{F E R}\right)$ chooses the MCS with the highest spectral efficiency satisfying the condition $f e r_{m, s}<t h_{F E R}$. MaxPHYThru, on the other hand, is a non-parametric LA scheme with the single purpose of maximizing PHY throughput disregarding the resulting FER and PER. The typical variant of MaxPHYThru which we denote by MaxPHYThru-FER chooses the MCS maximizing the term $\left(1-\mathrm{fer}_{m, s}\right) \log _{2}\left(V_{m}\right) R_{m}$ [66]. Since a successful packet transmission requires all building FEC blocks to be successfully transmitted, we also study a PER-based variant of MaxPHYThru denoted by MaxPHYThru-PER whose objective function is $\left(1-\right.$ per $\left._{m, s}\right) \log _{2}\left(V_{m}\right) R_{m}$. In Table 6 , the mean and the worst-case normalized aggregate TCP throughput performances $\boldsymbol{T}_{\boldsymbol{M}}$ and $\boldsymbol{T}_{W}$, respectively, of the QAWLA, TAGLA, TFER, and MaxPHYThru LA schemes are given for both the AWGN and the ITU-A channels. Threshold parameter $t h_{P E R}\left(t h_{F E R}\right)$ of TAGLA (TFER) is fixed to the value maximizing the average of $T_{M}$ for the AWGN and the ITU-A channels which is $0.0032(0.00013)$. TAGLA and TFER are based on the same principles except for a scaling of their threshold parameters and therefore they achieve almost the same performance. The loss-ignorant nature of both MaxPHYThru-FER and MaxPHYThru-PER schemes make them least likely candidates for TCP traffic which is wellknown for being sensitive to packet losses. As expected, MaxPHYThru-PER performs slightly better than MaxPHYThru-FER which assigns a lower penalty to transmission errors. Finally, we observe that QAWLA yields the best performance among the LA schemes studied in this paper.

Table 6 Normalized aggregate TCP throughput performance of various LA schemes

\begin{tabular}{llllll}
\hline & \multicolumn{2}{l}{ AWGN } & & \multicolumn{1}{l}{ ITU-A } \\
\cline { 2 - 3 } & $T_{M}$ & $T_{W}$ & & $T_{M}$ & $T_{W}$ \\
\hline QAWLA(0.05, 100, 20) & 0.99 & 0.92 & & 0.97 & 0.85 \\
TAGLA(0.0032) & 0.97 & 0.67 & & 0.89 & 0.52 \\
TFER(0.00013) & 0.97 & 0.67 & & 0.89 & 0.52 \\
MaxPHYThru-PER & 0.79 & 0.02 & & 0.43 & 0.04 \\
MaxPHYThru-FER & 0.51 & 0.00 & & 0.17 & 0.00 \\
\hline
\end{tabular}

\section{Conclusions}

A novel queue-aware link adaptation mechanism is proposed for wireless links carrying long-lived TCP flows and which are controlled by AQM buffer management. This proposed cross-layer mechanism is based on the choice of a different modulation and coding scheme depending on whether the queue occupancy is above or below a certain threshold at the epoch of packet transmission. A novel fixed-point analytical model is developed to accommodate discontinuous wireless packet loss rate and queue service rate of this dual-regime queuing system which is validated with extensive ns-3 simulations. Using the proposed analytical model, we show significant TCP throughput improvement with queue awareness for link adaptation. Such throughput improvement is shown to exist even when the channel statistics are not precisely known. Investigating the performance of the proposed QAWLA scheme for PHY technologies other than IEEE 802.16 such as LTE, consideration of the use of HARQ/ARQ techniques at the PHY, use of multiple thresholds as opposed to one single threshold of the DRWL framework, and the employment of queue-awareness in link adaptation of point-to-multipoint wireless systems, such as cellular networks, are left for future research.

\section{Competing interests}

The authors declare that they have no competing interests.

Received: 21 February 2015 Accepted: 19 October 2015 Published online: 23 November 2015

\section{References}

1. RKP Mok, EWW Chan, RKC Chang, in Integrated Network Management (IM), 2011 IFIP/IEEE International Symposium On. Measuring the quality of experience of HTTP video streaming (IEEE, USA, 2011), pp. 485-492

2. D Lee, BE Carpenter, N Brownlee, in Internet Monitoring and Protection (ICIMP), 2010 Fifth International Conference On. Observations of UDP to TCP ratio and port numbers (IEEE, USA, 2010), pp. 99-104

3. C Lee, D Lee, S Moon, in Passive and Active Measurement Lecture Notes in Computer Science, ed. by N Taft, F Ricciato. Unmasking the growing UDP traffic in a campus network, vol. 7192 (Springer, Berlin Heidelberg, 2012), pp. $1-10$

4. B Braden, D Clark, J Crowcroft, B Davie, S Deering, D Estrin, S Floyd, V Jacobson, G Minshall, C Partridge, L Peterson, K Ramakrishnan, S Shenker, J Wroclawski, L Zhang, Recommendations on queue management and congestion avoidance in the Internet. RFC 2309. (RFC Editor, 1998). http:// tools.ietf.org/html/rfc2309, access date 29 October 2015

5. S Floyd, $V$ Jacobson, Random early detection gateways for congestion avoidance. IEEE/ACM Trans. Networking. 1(4), 397-413 (1993)

6. ES Hashem, Analysis of random drop for gateway congestion control. PhD thesis, Massachusetts Institute of Technology (1989)

7. S Athuraliya, SH Low, VH Li, Q Yin, REM: active queue management. IEEE Netw. 15(3), 48-53 (2001)

8. M Hassan, R Jain, High Performance TCP/IP Networking: Concepts, Issues, and Solutions. (Pearson, US, 2004)

9. R Adams, Active queue management: a survey. IEEE Commun. Surv. Tutorials. 15(3), 1425-1476 (2013)

10. H Balakrishnan, VN Padmanabhan, S Seshan, RH Katz, A comparison of mechanisms for improving TCP performance over wireless links. IEEE/ACM Trans. Networking. 5(6), 756-769 (1997)

11. S Catreux, V Erceg, D Gesbert, RW Heath, Adaptive modulation and MIMO coding for broadband wireless data networks. IEEE Commun. Mag. 40(6), 108-115 (2002) 
12. ST Chung, AJ Goldsmith, Degrees of freedom in adaptive modulation: a unified view. IEEE Trans. Commun. 49(9), 1561-1571 (2001)

13. J Yang, N Tin, AK Khandani, in Adaptive modulation and coding in $3 G$ wireless systems. Vehicular Technology Conference, 2002. Proceedings. VTC 2002-Fall. 2002 IEEE 56th, vol. 1, (2002), pp. 544-5481

14. H Chen, HCB Chan, C-K Chan, VCM Leung, QoS-based cross-layer scheduling for wireless multimedia transmissions with adaptive modulation and coding. IEEE Trans. Commun. 61(11), 4526-4538 (2013)

15. K-C Go, J-H Kim, Application-aware MCS level selection method for cross-layered retransmission scheme. IEEE Commun. Lett. 18(2), 209-212 (2014)

16. Q Liu, S Zhou, GB Giannakis, Queuing with adaptive modulation and coding over wireless links: cross-layer analysis and design. IEEE Trans. Wirel. Commun. 4(3), 1142-1153 (2005)

17. D Moltchanov, A study of TCP performance in wireless environment using fixed-point approximation. Comput. Netw. 56(4), 1263-1285 (2012)

18. A De Vendictis, A Baiocchi, in Modeling a mixed TCP Vegas and TCP Reno scenario, ed. by E Gregori, M Conti, A Campbell, G Omidyar, and M Zukerman. NETWORKING 2002: Networking Technologies, Services, and Protocols; Performance of Computer and Communication Networks; Mobile and Wireless Communications. Lecture Notes in Computer Science, vol. 2345 (Springer, Berlin Heidelberg, 2002), pp. 612-623

19. V Kokshenev, S Suschenko, in Analytical model of the TCP Reno congestion control procedure through a discrete-time Markov chain, ed. by V Vishnevsky, D Kozyrev, and A Larionov. Distributed Computer and Communication Networks. Communications in Computer and Information Science, vol. 279 (Springer International Publishing, 2014), pp. 124-135

20. V Jacobson, Congestion avoidance and control. SIGCOMM Comput. Commun. Rev. 18(4), 314-329 (1988)

21. V Jacobson, Modified TCP congestion avoidance algorithm. Technical report (1990). end2end-interest mailing list. ftp://ftp.isi.edu/end2end/ end2end-interest-1990.mail, access date 29 October 2015

22. K Tan, J Song, Q Zhang, M Sridharan, in INFOCOM 2006. 25th IEEE International Conference on Computer Communications. Proceedings. A compound TCP approach for high-speed and long distance networks (IEEE, USA, 2006), pp. 1-12

23. $\mathrm{SHa}$, I Rhee, $L \mathrm{Xu}, \mathrm{CUBIC}$ : A New TCP-friendly high-speed TCP variant. SIGOPS Oper. Syst. Rev. 42(5), 64-74 (2008)

24. P Yang, J Shao, $W$ Luo, L X Xu, J Deogun, $Y$ Lu, TCP congestion avoidance algorithm identification. IEEE/ACM Trans. Networking. 22(4), 1311-1324 (2014)

25. G Martorell, F Riera-Palou, G Femenias, Cross-layer fast link adaptation for MIMO-OFDM based WLANs. Wirel. Pers. Commun. 56(3), 599-609 (2011)

26. O Ozturk, N Akar, Workload-dependent queuing model of an AQMcontrolled wireless router with TCP traffic and its application to PER-based link adaptation. EURASIP J. Wirel. Commun. Netw. 67(1), 1-18 (2014)

27. Q Liu, S Zhou, GB Giannakis, in TCP performance in wireless access with adaptive modulation and coding. Communications, $2004 \mathrm{IEEE}$ International Conference On, vol. 7, (2004), pp. 3989-39937

28. H Jiang, C Dovrolis, Passive estimation of TCP round-trip times. SIGCOMM Comput. Commun. Rev. 32(3), 75-88 (2002)

29. J Huang, Z Niu, in Wireless Communications and Networking Conference, 2007.WCNC 2007. IEEE. Buffer-aware and traffic-dependent packet scheduling in wireless OFDM networks (IEEE, USA, 2007), pp. 1554-1558

30. Y Lin, G Yue, in Wireless Communications, Networking and Mobile Computing, 2008. WiCOM '08. 4th International Conference On. Channel-adapted and buffer-aware packet scheduling in LTE wireless communication system, (2008), pp. 1-4

31. D Niyato, E Hossain, Queue-aware uplink bandwidth allocation and rate control for polling service in IEEE 802.16 broadband wireless networks. IEEE Trans. Mob. Comput. 5(6), 668-679 (2006)

32. G Song, Y Li, LJ Cimini, Joint channel- and queue-aware scheduling for multiuser diversity in wireless OFDMA networks. IEEE Trans. Commun. 57(7), 2109-2121 (2009)

33. E Uysal-Biyikoglu, A El Gamal, On adaptive transmission for energy efficiency in wireless data networks. IEEE Trans. Inf. Theory. 50(12), 3081-3094 (2004)

34. X Li, X Dong, D Wu, in Military Communications Conference, 2008. MILCOM 2008. IEEE. Hierarchical queue-length-aware power control for delay-sensitive applications over wireless networks (IEEE, USA, 2008), pp. 1-6
35. EC Strinati, A De Domenico, L Herault, Green communications: an emerging challenge for mobile broadband communication networks. Journal of Green Engineering. 1(3), 267-301 (2011)

36. MI Salman, CK Ng, NK Noordin, BM Ali, A Sali, A self-configured link adaptation for green LTE downlink transmission. Transactions on Emerging Telecommunications Technologies. 26(2), 258-275 (2015)

37. X Li, X Dong, D Wu, On optimal power control for delay-constrained communication over fading channels. IEEE Trans. Inf. Theory. 57(6), 3371-3389 (2011)

38. X Ao, S Jiang, L Tang, in Communication Technology (ICCT), 201012 th IEEE International Conference On. Traffic-aware active link rate adaptation via power control for multi-hop multi-rate 802.11 networks (IEEE, USA, 2010), pp. 1255-1259

39. M Chiang, J Bell, in Balancing supply and demand of bandwidth in wireless cellular networks: Utility maximization over powers and rates. INFOCOM 2004. Twenty-third AnnualJoint Conference of the IEEE Computer and Communications Societies, vol. 4 (IEEE, USA, 2004), pp. 2800-28114

40. M Chiang, Balancing transport and physical layers in wireless multihop networks: jointly optimal congestion control and power control. IEEE J. Sel. Areas Commun. 23(1), 104-116 (2005)

41. TMC Chu, H Phan, H-J Zepernick, in Communications (ICC), 2014 IEEE International Conference On. Adaptive modulation and coding with queue awareness in cognitive incremental decode-and-forward relay networks, (2014), pp. 1453-1459

42. J Padhye, V Firoiu, D Towsley, J Kurose, Modeling TCP throughput: a simple model and its empirical validation. SIGCOMM Comput. Commun. Rev. 28(4), 303-314 (1998)

43. T Bu, D Towsley, Fixed point approximations for TCP behavior in an AQM network. SIGMETRICS Perform. Eval. Rev. 29(1), 216-225 (2001)

44. S Floyd, Recommendation on using the "Gentle" variant of RED. Technical report (2000). http://www.icir.org/floyd/red/gentle.html, access date 29 October 2015

45. S Athuraliya, SH Low, VH Li, Q Yin, REM: Active queue management. IEEE Netw. 15(3), 48-53 (2001)

46. H Balakrishnan, VN Padmanabhan, G Fairhurst, M Sooriyabandara, TCP performance implications of network path asymmetry. RFC 3449. (RFC Editor, 2002). http://www.rfc-editor.org/rfc/rfc3449.txt, access date 29 October 2015

47. TJ Speight, Efficient TCP ACK Prioritization in Wireless Networks. US20080080464 A1 (2008). Patent US20080080464

48. Ns-3 (2015). https://www.nsnam.org/, access date 29 October 2015

49. V Paxson, M Allman, J Chu, M Sargent, Computing TCP's retransmission timer. RFC 6298. (RFC Editor, 2011). http://www.rfc-editor.org/rfc/rfc6298. txt, access date 29 October 2015

50. IEEE standard for local and metropolitan area networks part 16: air interface for broadband wireless access systems, 1-2080 (2009). IEEE Std 802.16-2009 (Revision of IEEE Std 802.16-2004)

51. DT Chen, in IEEE Radio and Wireless Symposium 2007. On the analysis of using 802.16e WiMAX for point-to-point wireless backhaul, (2007), pp. 507-510

52. JARP de Carvalho, H Veiga, P Gomes, AD Reis, R Costa, in Signal Processing and Information Technology. ISSPIT 2008. IEEE International Symposium On. A contribution to experimental performance evaluation of point-to-point WiMAX links (IEEE, USA, 2008), pp. 150-153

53. PTP Solutions Guide Motorola Fixed Point-to-point Wireless Bridges. http://www.motorolasolutions.com/, access date 29 October 2015

54. RDL-3000 System. http://rdlcom.com/, access date 29 October 2015

55. Harris Falcon III ${ }^{\oplus F-7800 W}$. http://harris.com/, access date 29 October 2015

56. Coded Modulation Library (CML). http://www.iterativesolutions.com/, access date 29 October 2015

57. Guidelines for evaluation of radio transmission technologies for IMT-2000 (1997). Recommendation ITU-R M.1225

58. WiMAX Forum ${ }^{\oplus}$ mobile system profile: Release 1.5 TDD specific part Technical report, WiMAX Forum ${ }^{\oplus}$ (Mar 2009)

59. P Romirer-Maierhofer, F Ricciato, A D'Alconzo, R Franzan, W Karner, in Network-wide measurements of TCP RTT in 3G, ed. by M Papadopouli, P Owezarski, and A Pras. Traffic Monitoring and Analysis. Lecture Notes in Computer Science, vol. 5537 (Springer, Berlin Heidelberg, 2009), pp. 17-25

60. S Cen, PC Cosman, GM Voelker, End-to-end differentiation of congestion and wireless losses. IEEE/ACM Trans. Netw. 11(5), 703-717 (2003) 
61. S Floyd, E Kohler, Internet research needs better models. SIGCOMM Comput. Commun. Rev. 33(1), 29-34 (2003)

62. J Hespanha, S Bohacek, K Obraczka, J Lee, in Hybrid modeling of TCP congestion control, ed. by M Di Benedetto, A Sangiovanni-Vincentelli. Hybrid systems: computation and control. Lecture Notes in Computer Science, vol. 2034 (Springer, Berlin Heidelberg, 2001), pp. 291-304

63. G Carneiro, P Fortuna, M Ricardo, in FlowMonitor: A network monitoring framework for the network simulator 3 (NS-3). Proceedings of the Fourth International ICST Conference on Performance Evaluation Methodologies and Tools. VALUETOOLS '09 (ICST (Institute for Computer Sciences, Social-Informatics and Telecommunications Engineering) Brussels, Belgium, 2009), pp. 1-1110

64. G Ciccarese, M De Blasi, P Marra, C Palazzo, L Patrono, An algorithm for controlling packet size in IEEE 802.16e networks. Comput. Netw. 55(13), 2873-2885 (2011)

65. J Francis, NB Mehta, EESM-based link adaptation in point-to-point and multi-cell OFDM systems: Modeling and analysis. IEEE Trans. Wirel. Commun. 13(1), 407-417 (2014)

66. D Takeda, YC Chow, P Strauch, H Tsurumi, in Threshold controlling scheme for adaptive modulation and coding system. Personal, Indoor and Mobile Radio Communications, 2004. PIMRC 2004. 15th IEEE International Symposium On, vol. 2 (IEEE, USA, 2004), pp. 1351-1355

\section{Submit your manuscript to a SpringerOpen ${ }^{\mathcal{O}}$ journal and benefit from:}

- Convenient online submission

- Rigorous peer review

- Immediate publication on acceptance

- Open access: articles freely available online

- High visibility within the field

- Retaining the copyright to your article

Submit your next manuscript at $\boldsymbol{\wedge}$ springeropen.com 